\title{
Continuous dependence and error estimation for viscosity methods
}

\author{
Bernardo Cockburn \\ School of Mathematics, \\ University of Minnesota, \\ Minneapolis, Minnesota 55455, USA \\ E-mail: cockburn@math.umn.edu
}

In this paper, we review some ideas on continuous dependence results for the entropy solution of hyperbolic scalar conservation laws. They lead to a complete $L^{\infty}\left(L^{1}\right)$-approximation theory with which error estimates for numerical methods for this type of equation can be obtained. The approach we consider consists in obtaining continuous dependence results for the solutions of parabolic conservation laws and deducing from them the corresponding results for the entropy solution. This is a natural approach, as the entropy solution is nothing but the limit of solutions of parabolic scalar conservation laws as the viscosity coefficient goes to zero.

\section{CONTENTS}

1 Introduction 2

2 The main difficulty: the loss of well-posedness 4

3 Physically relevant solutions $\quad 9$

4 Continuous dependence for parabolic solutions 13

5 Robustness in the viscosity coefficient 18

6 Approximation theory for entropy solutions 21

7 Characterization of the entropy solution 24

8 Error estimates for the Engquist-Osher scheme 28

9 The historical evolution of some ideas 30

10 Concluding remarks and open problems $\quad 39$

References 40

Appendix: Proofs of some results 46 


\section{Introduction}

In this paper, we review some of the ideas on continuous dependence and error estimation for numerical methods for the Cauchy problem for the scalar hyperbolic conservation law

$$
\begin{aligned}
u_{t}+\nabla \cdot \mathbf{f}(u) & =r, \quad \\
u(t=0) & =u_{0}, \quad \text { in } \mathbb{R}^{d} \times(0, T), \\
u\left(\mathbb{R}^{d},\right. &
\end{aligned}
$$

that have been entertained during the last three decades.

It is well known that continuous dependence results are essential in order to have a mathematically sound approximation theory upon which the devising and analysis of numerical methods could be based. To illustrate this point, let us assume that we are interested in finding an approximation $v$, given by the numerical scheme $\mathbb{L}_{h}(v)=0$, to $u$ such that

$$
\|u-v\| \leq \text { tol, }
$$

where $\|\cdot\|$ is some norm and tol a given positive parameter, and let us show how to use a continuous dependence result for the solution $u$, with respect to the right-hand side $r$, to study this problem. If $u_{i}$ is the solution of the above Cauchy problem with $r=r_{i}, i=1,2$, the estimate

$$
\left\|u_{1}-u_{2}\right\| \leq \Phi\left(r_{1}-r_{2}\right)
$$

would allow us to compare the solution $u$ with any other function $v$ by simply setting $u_{1}=u, u_{2}=v$ and

$$
r_{2}=v_{t}+\nabla \cdot \mathbf{f}(v) .
$$

Indeed, in this case, we would obtain

$$
\|u-v\| \leq \Phi(-R(v)),
$$

where

$$
R(v)=v_{t}+\nabla \cdot \mathbf{f}(v)-r
$$

is nothing but the residual of $v$. Thus, to achieve our goal, it is enough to find $v$ such that

$$
\Phi(-R(v)) \leq \text { tol. }
$$

We see that the study of how to find the approximation $v$ becomes the study of how to minimize the nonlinear functional $\Phi(-R(v))$ in an optimal way with the restriction that the function $v$ is determined by the numerical scheme $\mathbb{L}_{h}(v)=0$. This is in fact one of the most important problems in modern computational partial differential equations. 
Unfortunately, the main difficulty in dealing with the Cauchy problem for the scalar hyperbolic conservation law is that its solution is not unique, as we will show in Section 2. Given that every physically relevant phenomenon should be modelled by a mathematically well-posed problem, we might then wonder how is it possible that scalar hyperbolic conservation laws are considered at all? The answer is that these equations are obtained from a well-posed problem by formally neglecting terms modelling effects considered to be non-dominant. A typical example occurs when, in the convection-diffusion Cauchy problem,

$$
\begin{aligned}
\left(u_{\nu}\right)_{t}+\nabla \cdot \mathbf{f}\left(u_{\nu}\right)-\nu \Delta u_{\nu} & =r, & & \text { in } \mathbb{R}^{d} \times(0, T), \\
u_{\nu}(t=0) & =u_{0}, & & \text { on } \mathbb{R}^{d},
\end{aligned}
$$

the term $\nu \Delta u_{\nu}$ modelling the viscosity effects is neglected because diffusion is considered to be unimportant. In Sections 2 and 3, we elaborate on this point.

Fortunately, this formal procedure can still be rendered meaningful if we obtain continuous dependence results for the well-posed convection-diffusion problem which do not break down when we let the viscosity coefficient tend to zero: see Sections 4 and 5. Indeed, armed with such results, it is possible to achieve the following.

(i) Prove the existence and uniqueness of the function $u=\lim _{\nu \downarrow 0} u_{\nu}$ which is thus considered to be the physically relevant solution of the scalar hyperbolic conservation law: see Section 5 .

(ii) Prove that these continuous dependence results also hold for this solution: see Section 5 .

(iii) Characterize this solution: see Section 7.

(iv) Construct a complete approximation theory for this type of solution: see Sections 6 and 8.

In Section 9 we discuss the evolution of some ideas leading to this approach, and in Section 10 we end with some concluding remarks. In the Appendix, we sketch the proofs of the main results.

The above-mentioned approach has recently been proposed for HamiltonJacobi equations and might also be used to study strongly degenerate scalar equations and viscosity solutions of nonlinear degenerate parabolic equations. To apply such an approach to hyperbolic systems is certainly a Herculean task, especially in view of the now classical result by Rauch (1986), which states that "for most non-scalar systems of conservation laws in dimension greater than one, one does not have BV estimates of the form

$$
\|\nabla u(t)\|_{L^{1}} \leq F\left(\|\nabla u(0)\|_{L^{1}}\right) . "
$$


Here $F$ is a continuous function such that $F(0)=0$ and $F$ is Lipschitz at 0 . On the other hand, in some cases, we might be closer to achieving that goal than would have been expected only a few years ago, given the recent developments in the theory of hyperbolic systems: see Bianchini and Bressan (2001).

Let us mention that the reader interested in the theoretical aspects of hyperbolic problems should consult the books by Bressan (2000), Dafermos (2000), Lax (1972), Liu (1997), Serre (1999, 2000) and Witham (1974). The reader interested in the numerics for this kind of problem should consult Barth and Deconink (1999), Cockburn and Shu (2001), Cockburn, Karniadakis and Shu (2000), Eymard, Gallouët and Herbin (2000), Godlewski and Raviart (1996), Holden and Risebro (2002), Johnson (1998), Kröner (1997), Kröner, Ohlberger and Rohde (1999), LeVeque (1990), Lucier (2003), Tadmor (1998), Shu (1998), and Toro (1997).

An elegant theory of continuous dependence in a negative-order norm for the physically relevant solutions of the conservation law

$$
u_{t}+(f(u))_{x}=0
$$

when $f$ is strictly convex, is reviewed in Tadmor (1998); here we are concerned with general nonlinearities $\mathbf{f}$ in a multi-dimensional setting. Finally, let us point out that, although parts of the material presented in this review can be found in some of the above-mentioned references, in particular in Lucier (2003), the approach proposed here and many of its main results cannot be found in any of them.

\section{The main difficulty: the loss of well-posedness}

In many instances, nonlinear hyperbolic problems arise from a well-posed problem by neglecting the terms that capture physical phenomena considered to be non-dominant; unfortunately, this renders the problem ill-posed. This loss of well-posedness is the main difficulty we face when dealing with nonlinear hyperbolic problems.

In this section, we illustrate this phenomenon in the setting of a simple traffic flow model. First, we display a well-posed parabolic model; the 'driver's awareness of the conditions ahead' giving a parabolic character to the model. We then argue that, if the driver's awareness is negligible, a scalar nonlinear hyperbolic conservation law is obtained, which gives rise to an ill-posed problem. We show that this happens because, although the neglected physical phenomenon can be correctly considered to be unimportant in most parts of the domain, it is still crucial in small parts of the domain, namely, near a strong variation of the density of cars. Thus, the removal of this essential physical information has, not surprisingly, disastrous consequences. 


\subsection{The model}

If $\rho$ represents the density of cars on a highway and $v$ represents the flow velocity, the conservation of mass is

$$
\rho_{t}+(\rho v)_{x}=0 \text {. }
$$

Following Witham (1974), we take the flow velocity to be the following function of the density of cars $\rho$ and of its gradient $\rho_{x}$ :

$$
\rho v=f(\rho)-\nu \rho_{x} .
$$

It is reasonable to assume that the so-called density flow $f(\rho)$ is of the form $\rho V(\rho)$, where $\rho \mapsto V(\rho)$ is a decreasing mapping which, for a given density, say $\rho^{\star}$, is equal to zero: this corresponds to the situation in which the cars are bumper to bumper. Maybe the simplest function $V$ satisfying these properties is

$$
V(\rho)=v_{\max }\left(1-\frac{\rho}{\rho^{\star}}\right),
$$

where $v_{\max }$ represents the maximum velocity. The term $\nu \rho_{x}$ models the 'awareness of conditions ahead', since, when we perceive a high increase in the density of cars ahead, we try to decelerate to avoid a potentially dangerous situation. With this choice of flow velocity, our conservation law becomes the parabolic equation

$$
\rho_{t}+(f(\rho))_{x}-\nu \rho_{x x}=0
$$

which, after the change of variables $t:=t / T, x:=x / L$, where $T=L / v_{\max }$, reads

$$
\phi_{t}+(f(\phi))_{x}-\epsilon \phi_{x x}=0
$$

where $\phi=\rho / \rho^{\star}$ and

$$
f(\phi):=\phi(1-\phi), \quad \epsilon=\frac{\nu}{L v_{\max }} .
$$

The dimensionless parameter $\epsilon$ measures the ability of the driver to react properly to a high concentration of cars at a distance $L$ when the maximum speed is $v_{\max }$. Clearly, if $\epsilon$ is very small, it seems reasonable to formally drop the second-order term from our model and consider instead the scalar nonlinear conservation law

$$
\phi_{t}+(f(\phi))_{x}=0
$$

Next, we explore the consequences of this formal procedure.

\subsection{Travelling waves}

The simplest way to do that is to consider travelling wave solutions of (2.1) and study their limit as the parameter $\epsilon$ tends to zero. 

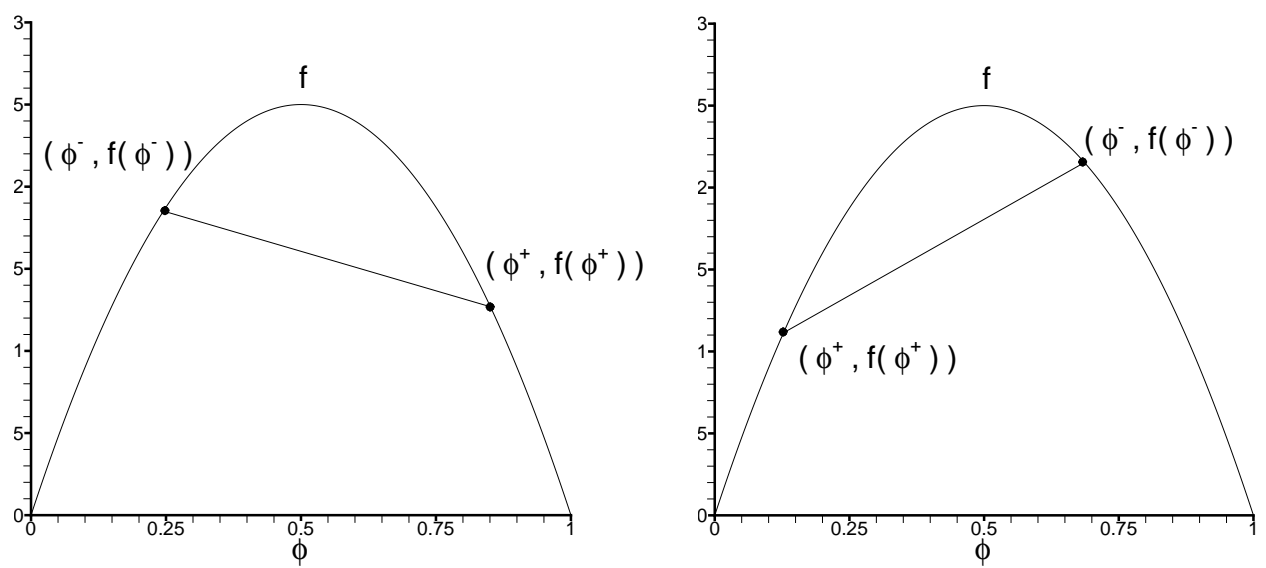

Figure 2.1. The condition for existence of a travelling wave joining $\phi^{-}$to $\phi^{+}$: satisfied (left) and not satisfied (right).

Travelling wave solutions of the parabolic conservation law (2.1) are solutions of the form $\phi(x, t)=\varphi((x-c t) / \epsilon))$ that satisfy the conditions $\lim _{z \rightarrow \infty^{ \pm}} \varphi(z)=\phi^{ \pm}$and $\lim _{z \rightarrow \infty^{ \pm}} \varphi^{\prime}(z)=0$. Inserting this expression for $\phi$ in the conservation law (2.1), and integrating once, we get

$$
\varphi^{\prime}=f(\varphi)-\mathcal{L}(\varphi),
$$

where the function $\mathcal{L}$ and the velocity $c$ of the travelling wave are given by

$$
\mathcal{L}(\varphi)=f\left(\phi^{+}\right)+c\left(\varphi-\phi^{+}\right), \quad c=\frac{f\left(\phi^{+}\right)-f\left(\phi^{-}\right)}{\phi^{+}-\phi^{-}} .
$$

Note that $\mathcal{L}$ is nothing but the linear function that coincides with $f$ at $\phi^{ \pm}$.

It is easy to see that there is a solution of the above ordinary differential equation if and only if

$$
\begin{array}{ll}
(f(\varphi)-\mathcal{L}(\varphi))>0 & \text { for } \phi^{+}>\phi>\phi^{-}, \\
(f(\varphi)-\mathcal{L}(\varphi))<0 & \text { for } \phi^{+}<\phi<\phi^{-},
\end{array}
$$

and if the conditions at infinity are satisfied, that is, if

$$
\lim _{\varphi \rightarrow \phi^{ \pm}} \int_{\varphi(0)}^{\varphi} \frac{\mathrm{d} \theta}{f(\theta)-\mathcal{L}(\theta)}= \pm \infty .
$$

Note that condition (2.6) states that the graph of $f$ on the interval $\left(\phi^{-}, \phi^{+}\right)$(resp., $\left.\left(\phi^{+}, \phi^{-}\right)\right)$lies strictly above (resp., below) the straight line joining the points $\left(\phi^{ \pm}, f\left(\phi^{ \pm}\right)\right)$. Thus, for the concave nonlinearity $f$ given by $(2.2)$, it becomes simply $\phi^{-}<\phi^{+}$; see Figure 2.1 .

We summarize these findings in the following result. 

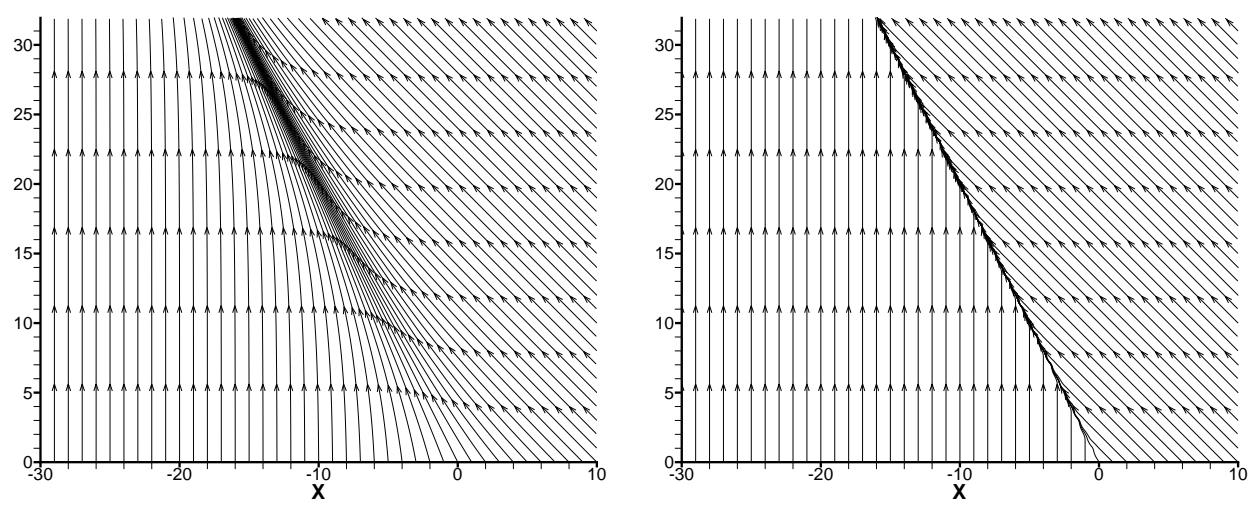

Figure 2.2. The curves $\left\{(x(t), t): \dot{x}=f^{\prime}\left(\varphi\left(\frac{x-c t}{\epsilon}\right)\right)\right\}$ for $\epsilon=1$ (left) and $\epsilon=0.1$ (right) for the case $\phi^{-}=1 / 2$ and $\phi^{+}=1$.

Theorem 2.1. A travelling wave solution of the parabolic conservation law (2.1) exists if and only if conditions (2.6) and (2.7) are satisfied.

Next, we study what happens when we let the coefficient $\epsilon$ tend to zero. We begin by noting that the limit of the travelling wave solution is

$$
\hat{\phi}(x, t)=\phi_{0}(x-c t), \quad \phi_{0}(x)=\lim _{\epsilon \downarrow 0} \varphi(x / \epsilon)= \begin{cases}\phi^{+} & \text {if } x>0, \\ \phi^{-} & \text {if } x<0 .\end{cases}
$$

We illustrate this passage to the limit in Figure 2.2.

To see what Cauchy problem this limit is a solution of, we proceed as follows. Multiplying the parabolic conservation law (2.1) by an arbitrary function $\eta$ in $\mathcal{C}_{0}^{\infty}(\mathbb{R} \times[0, T))$, the set of infinitely differentiable functions of compact support on $\mathbb{R} \times[0, T)$, integrating over the strip $\mathbb{R} \times[0, T)$ and integrating by parts, we obtain

$$
\int_{0}^{T} \int_{\mathbb{R}}\left(\phi(x, t) \eta(x, t)_{t}+f(\phi(x, t)) \eta(x, t)_{x}\right) \mathrm{d} x \mathrm{~d} t+\int_{\mathbb{R}} \phi(x, 0) \eta(x, 0) \mathrm{d} x+\Psi=0,
$$

where $\Psi$ is defined by

$$
\Psi=\epsilon \int_{0}^{T} \int_{\mathbb{R}} \phi(x, t) \eta(x, t)_{x x} \mathrm{~d} x \mathrm{~d} t .
$$

Taking $\phi(x, t)=\varphi\left(\frac{x-c t}{\epsilon}\right)$ and letting $\epsilon$ tend to zero, we obtain that $\hat{\phi}$ satisfies

$$
\int_{0}^{T} \int_{\mathbb{R}}\left(\hat{\phi}(x, t) \eta(x, t)_{t}+f(\hat{\phi}(x, t)) \eta(x, t)_{x}\right) \mathrm{d} x \mathrm{~d} t+\int_{\mathbb{R}} \phi_{0}(x) \eta(x, 0) \mathrm{d} x=0,
$$

for every function $\eta$ in $\mathcal{C}_{0}^{\infty}(\mathbb{R} \times[0, T))$. Any such function $\hat{\phi}$ is said to be a 

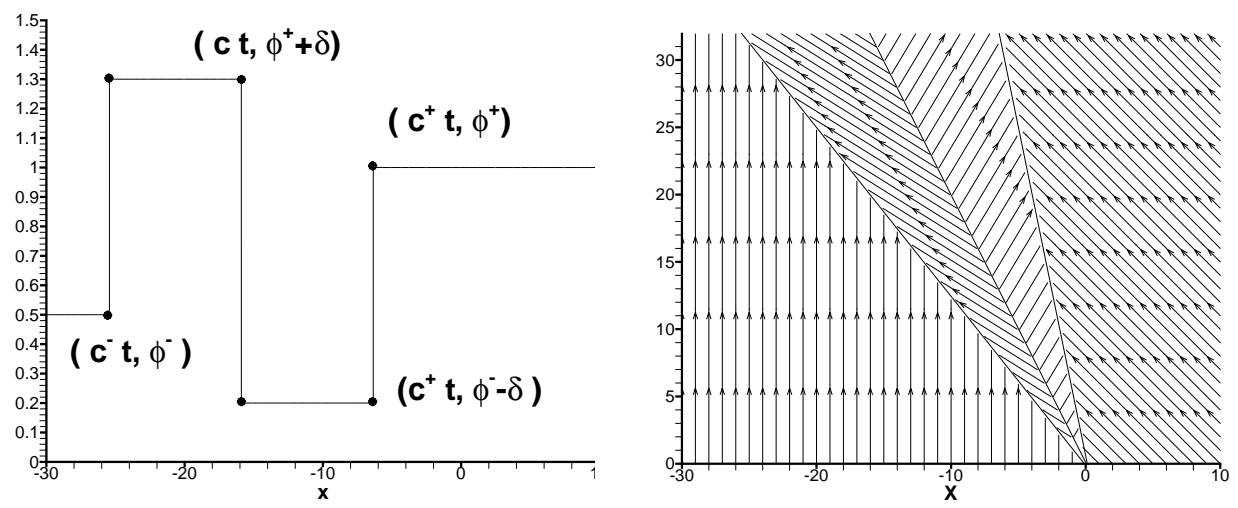

Figure 2.3. The weak solution $\phi^{\delta}(\cdot, t)$ (left) and the curves $(x(t), t)$ (right) for the case $\phi^{-}=1 / 2, \phi^{+}=1$ and $\delta=0.3$.

weak solution of the hyperbolic conservation law

$$
\phi_{t}+(f(\phi))_{x}=0
$$

with initial data $\phi_{0}$. This fact seems to indicate that dropping the secondorder term $\epsilon \phi_{x x}$ from the parabolic conservation law (2.1) is actually mathematically justified. However, it is possible to construct infinitely many weak solutions of the hyperbolic conservation law which satisfy the same initial condition satisfied by $\hat{\phi}$.

Indeed, it is easy to verify that, for every value of the nonnegative parameter $\delta$, the following is a weak solution of the hyperbolic conservation law (2.3) with initial condition $\phi_{0}$ :

$$
\phi^{\delta}(x, t)= \begin{cases}\phi^{-} & \text {if } c^{-}<x / t \\ \phi^{+}+\delta & \text { if } c<x / t<c^{-} \\ \phi^{-}-\delta & \text { if } c^{+}<x / t<c \\ \phi^{+} & \text {if } x / t<c^{+}\end{cases}
$$

where $c=1-\left(\phi^{+}+\phi^{-}\right), c^{-}=c-\delta, c^{+}=c+\delta$; see in Figure 2.3 the above weak solution for $\phi^{-}=1 / 2, \phi^{+}=1$ and $\delta=0.3$.

Note that for $\delta=0$ we obtain the limit of the travelling wave solutions, $\hat{\phi}$. For $\delta>0$, however, we obtain weak solutions with three discontinuity curves. Two of them, namely $x / t=c^{-}$and $x / t=c^{+}$, can be obtained as the limit of travelling wave solutions of the parabolic conservation law (2.1), since the conditions for the existence of travelling waves given by Theorem 2.1 are satisfied. This not the case, however, for the discontinuity $x / t=c$. In other words, this discontinuity does not 'remember' anything about the physics captured in the modelling of the awareness of the conditions lying ahead. 
Thus, as claimed at the beginning of the section, dropping the secondorder term $\epsilon \phi_{x x}$ from the parabolic conservation law (2.1) results in the loss of the well-posedness of the Cauchy problem for the nonlinear hyperbolic equation (2.3).

\section{Physically relevant solutions}

It is thus clear that, since there might be infinitely many weak solutions of the scalar hyperbolic conservation law, it is important to be able to select the physically relevant solution. Of course, this notion can only be relative to an already existing model. We thus say that a weak solution of the hyperbolic conservation law (2.3) is physically relevant with respect to the well-posed model

$$
\left(\phi_{\omega}\right)_{t}+\left(f\left(\phi_{\omega}\right)\right)_{x}+L\left(\omega ; \phi_{\omega}\right)=0
$$

where $L(0 ; \cdot)=0$, if it is the limit of solutions $\phi_{\omega}$ as $\omega$ tends to zero.

The purpose of this section is to show that a weak solution of the hyperbolic conservation law (2.3) can be physically relevant with respect to two different well-posed models simultaneously, and that it can also be physically relevant with respect to one model but not another. We illustrate this phenomenon by using the travelling wave solutions introduced in the previous section.

This implies that, when dealing with hyperbolic conservation laws, we must specify the well-posed problem with respect to which the weak solutions are physically relevant. Only then we are going to be able to recover the well-posedness lost when the operator $L(\omega ; \cdot)$ is formally dropped from the equation.

\subsection{Two parabolic models}

We begin by displaying two examples of models that produce the same physically relevant solution as our original model (2.1). The first is

$$
\phi_{t}+(f(\phi))_{x}-\epsilon\left(\mathrm{A}(\phi) \phi_{x}\right)_{x}=0 .
$$

It is easy to see that the travelling wave solutions must satisfy

$$
\varphi^{\prime}=\frac{f(\varphi)-\mathcal{L}(\varphi)}{\mathrm{A}(\varphi)}
$$

and, if $\mathrm{A}(\phi) \geq \mathrm{A}_{0}>0$, Theorem 2.1 holds if condition (2.7) is replaced by

$$
\lim _{\varphi \rightarrow \phi^{ \pm}} \int_{\varphi(0)}^{\varphi} \frac{\mathrm{A}(\theta) \mathrm{d} \theta}{f(\theta)-\mathcal{L}(\theta)}= \pm \infty .
$$

As a consequence, the limit of the travelling wave as $\epsilon \downarrow 0$ coincides with the limit obtained with the original model. 
The second example is

$$
\phi_{t}+(f(\phi))_{x}-\epsilon\left(\mathrm{B}\left(\phi_{x}\right)\right)_{x}=0,
$$

where B is a strictly increasing function. Indeed, in this case, since the travelling wave solutions satisfy

$$
\varphi^{\prime}=\mathrm{B}^{-1}(f(\varphi)-\mathcal{L}(\varphi)),
$$

Theorem 2.1 holds if condition (2.7) is replaced by

$$
\lim _{\varphi \rightarrow \phi^{ \pm}} \int_{\varphi(0)}^{\varphi} \frac{\mathrm{d} \theta}{\mathrm{B}^{-1}(f(\theta)-\mathcal{L}(\theta))}= \pm \infty .
$$

Once again, the limit of the travelling wave as $\epsilon \downarrow 0$ coincides with the limit obtained with the original model.

\subsection{The modified Korteweg-deVries-Burgers model}

Let us now consider the model

$$
\phi_{t}+(f(\phi))_{x}-\epsilon \phi_{x x}-\omega \phi_{x x x}=0,
$$

where $f(\phi)=\phi^{3}$. In this case, the travelling wave solutions are solutions of the form $\phi(x, t)=\varphi((x-c t) / \epsilon)$ that satisfy the conditions $\lim _{z \rightarrow \infty^{ \pm}} \varphi(z)=$ $\phi^{ \pm}, \lim _{z \rightarrow \infty^{ \pm}} \varphi^{\prime}(z)=0$, and $\lim _{z \rightarrow \infty^{ \pm}} \varphi^{\prime \prime}(z)=0$. Hence, they are solutions of

$$
\varphi^{\prime}+\mathrm{r} \varphi^{\prime \prime}=f(\phi)-\mathcal{L}(\phi)
$$

where $r=\omega / \epsilon^{2}$, satisfying the above conditions at $\pm \infty$. Equivalently, they are orbits of the dynamical system

$$
\frac{\mathrm{d}}{\mathrm{d} t}\left(\begin{array}{c}
\varphi \\
\eta
\end{array}\right)=\left(\begin{array}{c}
\eta \\
\frac{1}{\mathrm{r}}(f(\varphi)-\mathcal{L}(\varphi)-\eta)
\end{array}\right),
$$

that connect the equilibrium points $\left(\phi^{ \pm}, 0\right)$. We can immediately see that the conditions for these orbits to exist might be different from the conditions for the existence of travelling waves given by Theorem 2.1.

Jacobs, McKinney and Shearer (1995) considered the case $f(\phi)=\phi^{3}$. They showed that, when $\epsilon>0$ and $\omega<0$, the travelling wave solutions exist if and only if the travelling wave solutions of

$$
\phi_{t}+(f(\phi))_{x}-\epsilon \phi_{x x}=0
$$

exist. This implies that a weak solution is physically relevant with respect to both models at the same time. However, this is no longer true if $\omega>0$. Indeed, it was shown that there are travelling wave solutions depending on the ratio $r=\epsilon^{2} / \omega$ that are not travelling wave solutions of the original model. Thus a weak solution of the hyperbolic conservation law (2.3) can be physically relevant with respect to one model and not the other. Moreover, 
since the travelling wave solutions of the model under consideration depend on the ratio $r$, a weak solution of (2.3) might be physically relevant with respect to the model for one value or but not another.

\subsection{Phase transitions in solids}

A similar, but more complicated, situation appears in the so-called viscositycapillarity model for phase transitions in van der Waal fluids proposed by Truskinovsky (1982) and Slemrod (1984). Such a model reads

$$
\begin{aligned}
\gamma_{t} & =v_{x} \\
v_{t} & =(\sigma(\gamma))_{x}+\nu v_{x x}-\lambda \gamma_{x x x}
\end{aligned}
$$

where $v$ is the velocity, $\gamma$ the strain, and $\sigma$ the stress; the parameter $\nu$ is the viscosity and the parameter $\lambda$ the capillarity. Some solids admit several types of crystals and this is reflected in the fact that $\sigma$ is not an increasing function of the strain. As a consequence, if we drop the information about the viscosity and capillarity effects, we obtain the system

$$
\begin{aligned}
& \gamma_{t}=v_{x}, \\
& v_{t}=(\sigma(\gamma))_{x},
\end{aligned}
$$

which is not even hyperbolic, as it changes type as $\sigma^{\prime}$ changes sign.

The weak solutions of the above model that are physically relevant with respect to the viscosity-capillarity model has been studied in Abeyaratne and Knowles $(1991 a, 1991 b)$. Just as for the modified Korteweg-deVriesBurgers model, they have been shown to depend on the ratio $\lambda / \nu^{2}$. This fact has to be taken into consideration when devising numerical schemes that converge to those weak solutions; see Slemrod and Flaherty (1986), Shu (1992), Cockburn and Gau (1996) and Zhong, Hou and LeFloch (1996).

\subsection{Compressible fluid flow}

Perhaps the most widely known example of the situation under consideration is the case of Navier-Stokes for compressible fluid flow. Using classical tensor notation, they can be written as follows:

$$
\begin{aligned}
\rho_{t}+\left(\rho v_{j}\right)_{, j} & =0, \\
\left(\rho v_{i}\right)_{t}+\left(\rho v_{i} v_{j}-\sigma_{i j}\right)_{, j} & =f_{i}, \\
(\rho e)_{t}+\left(\rho e v_{j}-\sigma_{i j} v_{i}+q_{i}\right)_{, j} & =f_{i} v_{i},
\end{aligned}
$$

where $\rho$ is the density, $v$ the velocity, $e$ the internal energy, and $f$ the external body forces. The viscous stress $\sigma$ and the heat flux $q$ are given by

$$
\begin{aligned}
\sigma_{i j} & =\left(-p+\lambda v_{i, i}\right) \delta_{i j}+\mu\left(v_{i, j}+v_{j, i}\right), \\
q_{i} & =-\kappa T_{, i},
\end{aligned}
$$


where $p$ is the pressure and $T$ the temperature.

From the above equations, the following equation for the entropy $s$ can be obtained:

$$
(\rho s)_{t}+\left(\rho s v_{j}\right)_{, j}=\psi
$$

where

$$
\psi=\frac{\lambda}{T}\left(v_{i, i}\right)^{2}+\frac{\mu}{2 \lambda}\left(v_{i, j}+v_{j, i}-\frac{2}{3} v_{k, k} \delta_{i j}\right)^{2}+\frac{1}{T}\left(\kappa T_{, i}\right)_{, i} .
$$

If $\Omega$ is any domain on whose border we have $v_{i} n_{i}=0$ and $T_{, i} n_{i}=0$, we get

$$
\frac{\mathrm{d}}{\mathrm{d} t} \int_{\Omega} \rho s \mathrm{~d} x=\int_{\Omega} \Psi \mathrm{d} x
$$

where

$$
\Psi=\frac{\lambda}{T}\left(v_{i, i}\right)^{2}+\frac{\mu}{2 \lambda}\left(v_{i, j}+v_{j, i}-\frac{2}{3} v_{k, k} \delta_{i j}\right)^{2}+\frac{\kappa}{T^{2}}\left(T_{, i} T_{, i}\right) .
$$

By the second law of thermodynamics, the viscosity coefficients $\lambda$ and $\mu$, and the heat transfer coefficient $\kappa$ are positive and hence we have the so-called entropy condition

$$
\frac{\mathrm{d}}{\mathrm{d} t} \int_{\Omega} \rho s \mathrm{~d} x \geq 0
$$

If convection is the dominant feature of the flow and we formally neglect the effect of the viscosity and that of heat transfer, we obtain the nonlinear first-order system

$$
\begin{aligned}
\rho_{t}+\left(\rho v_{j}\right)_{, j} & =0 \\
\left(\rho v_{i}\right)_{t}+\left(\rho v_{i} v_{j}\right)_{, j}+p_{, i} & =f_{i}, \\
(\rho e)_{t}+\left(\rho e v_{j}-p v_{j}\right)_{, j} & =f_{i} v_{i},
\end{aligned}
$$

usually called the Euler equations. Only the weak solutions of these equations that satisfy the so-called entropy condition are considered to be physically relevant. For this reason, they are called the entropy solutions.

\subsection{Convection-diffusion equations}

It would have been ideal to work with the Navier-Stokes equations of compressible fluid flow and the corresponding Euler equations. Unfortunately, the link between these two equations, in particular concerning continuous dependence results, constitutes the subject of ongoing research.

In fact, such a link exists only for the scalar conservation law. For this reason, in the remainder of the paper, we are going to deal with the weak solutions of the hyperbolic conservation law

$$
u_{t}+\nabla \cdot \mathbf{f}(u)=r
$$


which are physically relevant with respect to the convection-diffusion model

$$
\left(u_{\nu}\right)_{t}+\nabla \cdot \mathbf{f}\left(u_{\nu}\right)-\nu \Delta u_{\nu}=r .
$$

In other words, we consider the weak solutions obtained by the so-called vanishing viscosity method, that is, as limits, when the viscosity coefficient $\nu$ tends to zero, of the solutions $u_{\nu}$ of the convection-diffusion equation.

Note that since the weak solutions of the scalar hyperbolic conservation law we are considering are obtained by the vanishing viscosity method, it would be quite natural to call them viscosity solutions. Moreover, this would emphasize the strong link between these solutions and the so-called viscosity solutions of the Hamilton-Jacobi equation

$$
u_{t}+H(\nabla u)=r
$$

see Crandall, Ishii and Lions (1992) and the references therein. These solutions, as we would expect, are obtained by the vanishing viscosity method, that is, as limits, when the viscosity coefficient $\nu$ tends to zero, of the solutions $u_{\nu}$ of

$$
\left(u_{\nu}\right)_{t}+H\left(\nabla u_{\nu}\right)-\nu \Delta u_{\nu}=r .
$$

However, the viscosity solutions for scalar hyperbolic problems are usually called entropy solutions (Lax 1972), as a mathematical generalization of what happens for the actual entropy solutions of compressible fluid flow.

\section{Continuous dependence for parabolic solutions}

In this section, we consider the initial value problem

$$
\begin{aligned}
u_{t}+\nabla \cdot \mathbf{f}(u)-\nu \Delta u & =r, & & \text { in } \mathbb{R}^{d} \times(0, T), \\
u(t=0) & =u_{0}, & & \text { on } \mathbb{R}^{d},
\end{aligned}
$$

and estimate the effect on the solution $u$ of changes in the initial data $u_{0}$ and the right-hand side $r$. In other words, we consider the solution of

$$
\begin{aligned}
v_{t}+\nabla \cdot \mathbf{f}(v)-\nu \Delta v & =s, & & \text { in } \mathbb{R}^{d} \times(0, T), \\
v(t=0) & =v_{0}, & & \text { on } \mathbb{R}^{d},
\end{aligned}
$$

and get a simple upper bound for the quantity

$$
|(u-v)(t)|_{U}:=\int_{\mathbb{R}^{d}} U(u(x, t)-v(x, t)) \mathrm{d} x,
$$

where each nonnegative function $U$ is determined to handle the effects of nonlinear convection suitably.

We show that such functions $U$ are associated with $L^{1}$-like norms and seminorms and that the upper bound we obtain gives rise to several powerful results, such as a priori bounds of the exact solutions; continuous 
dependence with respect to the initial data, the right-hand side and the nonlinearity $\mathbf{f}$; and to a posteriori error estimates. In spite of this, the estimate breaks down as the viscosity coefficient $\nu$ tends to zero. Fortunately, a modification of this approach does give a suitable continuous dependence result which, however, will be considered in the next section.

\subsection{Looking for suitable functions $U$}

In what follows, we assume that $u$ and $v$ are sufficiently smooth and decay to zero at infinity in such a way that all the formal steps we perform are justified. These properties do hold when the Lipschitz nonlinearity $\mathbf{f}$, the right-hand sides $r$ and $s$, and the initial data $u_{0}$ and $v_{0}$ are very smooth and decay to zero at infinity sufficiently fast; see Friedman (1964).

We begin with the following simple continuous dependence result.

Lemma 4.1. Let $u$ be the solution of (4.1) and (4.2), and let $v$ be the solution of (4.3) and (4.4). Let $U$ be any smooth function such that $U(0)=0$. Then

$$
|u(T)-v(T)|_{U}+\Theta_{U}=\left|u_{0}-v_{0}\right|_{U}+\int_{0}^{T} \int_{\mathbb{R}^{d}} U^{\prime}(u-v)(r-s) \mathrm{d} x \mathrm{~d} t
$$

where

$$
\Theta_{U}=\int_{0}^{T} \int_{\mathbb{R}^{d}}\left(Y_{\mathbf{f}, U}+Z_{\nu, U}\right) \mathrm{d} x \mathrm{~d} t
$$

and

$$
\begin{aligned}
& Y_{\mathbf{f}, U}=-U^{\prime \prime}(u-v)(\mathbf{f}(u)-\mathbf{f}(v)) \cdot \nabla(u-v), \\
& Z_{\nu, U}=\nu U^{\prime \prime}(u-v)|\nabla(u-v)|^{2} .
\end{aligned}
$$

Proof. From equations (4.1) and (4.3), we have

$$
(u-v)_{t}+\nabla \cdot(\mathbf{f}(u)-\mathbf{f}(v))-\nu \Delta(u-v)=r-s .
$$

Multiplying by $U^{\prime}(u-v)$, we obtain

$$
\begin{aligned}
(U(u-v))_{t}+\nabla \cdot\left(U^{\prime}(u-v)(\mathbf{f}(u)-\mathbf{f}(v))\right)+Y_{\mathbf{f}, U} \\
-\nu \Delta U(u-v)+Z_{\nu, U}=U^{\prime}(u-v)(r-s) .
\end{aligned}
$$

Integrating over $\mathbb{R}^{d} \times(0, T)$, we obtain the result. This completes the proof.

Note that if the convection is linear, that is, if $\mathbf{f}(u)=\mathbf{a} u$, we have that

$$
Y_{\mathbf{f}, U}=-U^{\prime \prime}(u-v)(u-v) \mathbf{a} \cdot \nabla(u-v),=\nabla \cdot\left(\mathbf{a} \int_{0}^{u-v} z U^{\prime \prime}(z) \mathrm{d} z\right),
$$

and so

$$
\Theta_{U}=\int_{0}^{T} \int_{\mathbb{R}^{d}}\left(Y_{\mathbf{f}, U}+Z_{\nu, U}\right) \mathrm{d} x \mathrm{~d} t=\int_{0}^{T} \int_{\mathbb{R}^{d}} Z_{\nu, U} \mathrm{~d} x \mathrm{~d} t
$$


This means that $\Theta_{U}$ is a nonnegative functional if $U$ is a convex function, as this would render nonnegative the term $Z_{\nu, U}$. This property of $U$ seems to be necessary to capture the dissipative nature of the Laplacian.

We would like to have $\Theta_{U}$ nonnegative even for a general nonlinearity $\mathbf{f}$. Hence we should find $U$ such that $e U^{\prime \prime}(e)=0$, as this ensures that $Y_{\mathbf{f}, U}=0$ given that $\mathbf{f}$ is Lipschitz. For such a function $U$, the functional $|\cdot|_{U}$ would then be perfectly tailored to handle nonlinear convection easily.

We can actually get such a function as the limit of a sequence of smooth functions $\left\{U_{\epsilon}\right\}_{\epsilon>0}$ such that $\lim _{\epsilon \mapsto 0} e U_{\epsilon}^{\prime \prime}(e)=0$. For example, we can take

$$
U_{\epsilon}(e)=a \Upsilon_{\epsilon}(e)+b \Upsilon_{\epsilon}(-e),
$$

where $a, b$ are nonnegative parameters, and

$$
\Upsilon_{\epsilon}(e)=\int_{0}^{e / \epsilon}(e-\epsilon s) \mu(s) \mathrm{d} s
$$

where $\mu$ is a smooth nonnegative function with support in $(0,1)$ and integral equal to one. Then we set

$$
U(e)=\lim _{\epsilon \downarrow 0} U_{\epsilon}(e) \quad \text { and } \quad U^{\prime}(e)=\lim _{\epsilon \downarrow 0} U_{\epsilon}^{\prime}(e) .
$$

For the special cases

$$
U(e)= \begin{cases}\max \{0, e\} & \text { for } a=1, b=0, \\ \max \{0,-e\} & \text { for } a=0, b=1, \\ |e| & \text { for } a=1, b=1\end{cases}
$$

Lemma 4.1 gives the following result.

Theorem 4.2. Let $u$ be the solution of (4.1) and (4.2) and let $v$ be the solution of (4.3) and (4.4). Let $U$ be given by (4.5). Then

$$
|u(T)-v(T)|_{U} \leq\left|u_{0}-v_{0}\right|_{U}+\int_{0}^{T}|(r-s)(t)|_{U} \mathrm{~d} t .
$$

Proof. The result follows from Lemma 4.1 and the fact that, for $U$ as in (4.5), we have $\lim _{\epsilon \downarrow 0} \Theta_{U_{\epsilon}} \geq 0$, and, for $U$ given by (4.5),

$$
\lim _{\epsilon \downarrow 0} \int_{0}^{T} \int_{\mathbb{R}^{d}} U_{\epsilon}^{\prime}(u-v)(r-s) \mathrm{d} x \mathrm{~d} t \leq \int_{0}^{T} \int_{\mathbb{R}^{d}} U(r-s) \mathrm{d} x \mathrm{~d} t .
$$

This completes the proof.

\subsection{Properties of the exact solutions}

As we see next, this powerful theorem gives rise to a priori estimates on the exact solutions; continuous dependence results on the initial data, the right-hand side, and the nonlinearity $\mathbf{f}$; and to a posteriori error estimates. 
To state these results, we use the following notation.

Definition. We write

$$
\|v\|_{L^{1}}=\|v\|_{L^{1}\left(\mathbb{R}^{d}\right)}, \quad|v|_{T V}=\sup _{\mathbf{w} \in \mathcal{C}_{0}^{\infty}\left(\mathbb{R}^{d}\right):\|\mathbf{w}\|_{L^{\infty}\left(\mathbb{R}^{d}\right)}=1} \int_{\mathbb{R}^{d}} v \nabla \cdot \mathbf{w} \mathrm{d} x,
$$

where $\|\mathbf{w}\|_{L^{\infty}(I)}=\max _{1 \leq i \leq d}\left\|\mathbf{w}_{i}\right\|_{L^{\infty}(I)}$. Note that, if $v$ is smooth,

$$
|w|_{T V}=\sum_{i=1}^{d}\left\|\partial_{x_{i}} w\right\|_{L^{1}}
$$

We shall also write

$$
\|w\|_{L^{\infty}(B)}=\sup _{t \in[0, T]}\|w(t)\|_{B}, \quad\|w\|_{L^{1}(B)}=\int_{0}^{T}\|w(t)\|_{B} \mathrm{~d} t,
$$

for $B=L^{1}$ and $B=T V$. Thus we shall speak of the $L^{1}\left(L^{1}\right)$ norm, for example.

Proposition 4.3. (A priori estimates) Let $u$ be the solution of (4.1) and (4.2). Then:

(i) the range of $u(T)$ is included in $[a(T), b(T)]$, where

$$
\begin{aligned}
& a(T)=\inf _{x \in \mathbb{R}^{d}} u_{0}(x)-\int_{0}^{T} \sup _{x \in \mathbb{R}^{d}} \max \{-r(x, t), 0\} \mathrm{d} t, \\
& b(T)=\sup _{x \in \mathbb{R}^{d}} u_{0}(x)+\int_{0}^{T} \sup _{x \in \mathbb{R}^{d}} \max \{r(x, t), 0\} \mathrm{d} t
\end{aligned}
$$

(ii) $\|u(T)\|_{T V} \leq\left\|u_{0}\right\|_{T V}+\|r\|_{L^{1}(T V)}$.

See the Appendix for a proof.

Proposition 4.4. (Continuous dependence) Now, let $v$ be the solution of (4.3) and (4.4). Then:

(i) if $s=r$ then $u_{0} \geq v_{0}$ implies $u(T) \geq v(T)$;

(ii) $\|u(T)-v(T)\|_{L^{1}} \leq\left\|u_{0}-v_{0}\right\|_{L^{1}}+\|r-s\|_{L^{1}\left(L^{1}\right)}$;

(iii) $\left\|u_{t}(T)\right\|_{L^{1}} \leq\|\mathbf{f}\|_{L^{\infty}(I)}\left|u_{0}\right|_{T V}+\nu\left\|\Delta u_{0}\right\|_{L^{1}}+\left\|r_{t}\right\|_{L^{1}\left(L^{1}\right)}$, where $I$ is the range of $u_{0}$.

Let $u_{i}$ be the solution of (4.1) and (4.2) with $\mathbf{f}=\mathbf{f}_{i}, i=1,2$. Then:

(iv) $\left\|u_{1}(T)-u_{2}(T)\right\|_{L^{1}} \leq\left\|\mathbf{f}_{1}^{\prime}-\mathbf{f}_{2}^{\prime}\right\|_{L^{\infty}(I)}\left\|u_{2}\right\|_{L^{1}(T V)}$, where $I$ is the range of $u_{2}$ on $\mathbb{R}^{d} \times(0, T)$.

See the Appendix for a proof. 
Let us emphasize that this result allows us to extend the notion of solution to initial data, right-hand sides, and nonlinearities $\mathbf{f}$ which need not be extremely smooth. To see this, note that, if $u_{n}$ is the smooth solution of (4.1) and (4.2) with $u_{0}=u_{0, n}$ and $r=r_{n}$ and the sequence $\left\{\left(u_{0, n}, r_{n}\right)\right\}$ converges strongly in $L^{1}\left(\mathbb{R}^{d}\right) \times L^{1}\left(0, T ; L^{1}\left(\mathbb{R}^{d}\right)\right)$ to a limit $\left(u_{0}^{\star}, r^{\star}\right)$, then (ii) implies that $\left\{u_{n}\right\}_{n \geq 0}$ is a Cauchy sequence in $\mathcal{C}^{0}\left(0, T ; L^{1}\left(\mathbb{R}^{d}\right)\right)$. Its limit, $u^{\star}$, is thus the physically relevant weak solution of (4.1) and (4.2) with initial data $u_{0}^{\star}$ and right-hand side $r^{\star}$. A similar argument holds when $u_{n}$ is the smooth solution of (4.1) and (4.2) with $\mathbf{f}=\mathbf{f}_{n}$.

Proposition 4.5. (A posteriori error estimate) Let $u$ be the solution of (4.1) and (4.2) and let $v$ be a smooth function whose residual

$$
R(v)=v_{t}+\nabla \cdot \mathbf{f}(v)-\nu \Delta v-r,
$$

belongs to $L^{1}\left(0, T ; L^{1}\left(\mathbb{R}^{d}\right)\right)$. Then, for $U$ given by (4.5),

$$
|u(T)-v(T)|_{U} \leq\left|u_{0}-v(0)\right|_{U}+\int_{0}^{T}|-R(v)(t)|_{U} \mathrm{~d} t .
$$

Proof. The result follows from Theorem 4.2 by simply taking $s=r+R(v)$.

This result allows us to estimate the quality of the approximation $v$ to $u$ without knowing the exact solution $u$; it is thus extremely useful in practical computations. In particular, when $U(e)=|e|$, we have the simple estimate

$$
\|u(T)-v(T)\|_{L^{1}} \leq\left\|u_{0}-v(0)\right\|_{L^{1}}+\|R(v)\|_{L^{1}\left(L^{1}\right)} .
$$

Unfortunately, this estimate is of no use as the viscosity coefficient tends to zero.

\subsection{Breakdown of the estimate as the viscosity tends to zero}

To see this, we begin by pointing out that, by Lemma 4.1, the quantity

$$
\left\|u_{0}-v(0)\right\|_{L^{1}}+\|R(v)\|_{L^{1}\left(L^{1}\right)}
$$

is in fact an upper bound for the sum

$$
\|u(T)-v(T)\|_{L^{1}}+\lim _{\epsilon \downarrow 0} \Theta_{U_{\epsilon}},
$$

where $U(e)=|e|$. Next we show that these two terms might be of significantly different sizes when the viscosity parameter $\nu$ tends to zero.

Let us consider the one-dimensional case, $d=1$, and the travelling wave solutions

$$
u(x, t)=\varphi((x-c t) / \nu), \quad v(x, t)=\varphi\left((x-c t) / \nu^{\prime}\right) .
$$


Then we have

$$
\lim _{\nu \downarrow 0} \lim _{\nu^{\prime} \downarrow 0}|u(T)-v(T)|_{U}=\lim _{\nu^{\prime} \downarrow 0} \lim _{\nu \downarrow 0}|u(T)-v(T)|_{U}=0 .
$$

On the other hand, we have

$$
\begin{aligned}
\lim _{\epsilon \downarrow 0} \Theta_{U_{\epsilon}} & =\lim _{\epsilon \downarrow 0} \int_{0}^{T} \int_{\mathbb{R}}\left(u_{x}-v_{x}\right)\left(U_{\epsilon}^{\prime}(u-v)\right)_{x} \mathrm{~d} x \mathrm{~d} t \\
& =2 \int_{0}^{T} \nu\left|\left(u_{x}-v_{x}\right)(c t, t)\right| \mathrm{d} t \\
& =2\left|1-\nu / \nu^{\prime}\right| \int_{0}^{T}|f(\varphi(0))-\mathcal{L}(\varphi(0))| \mathrm{d} t
\end{aligned}
$$

given that

$$
\eta w_{x}=\varphi^{\prime}=f(\varphi)-\mathcal{L}(\varphi)
$$

for $w(x, t)=\varphi((x-c t) / \eta)$, since $\varphi$ is a travelling wave solution; see (2.4). This implies

$$
\lim _{\nu \downarrow 0} \lim _{\epsilon \downarrow 0} \Theta_{U_{\epsilon}}=2 T|f(\varphi(0))-\mathcal{L}(\varphi(0))|>0,
$$

and

$$
\lim _{\nu^{\prime} \downarrow 0} \lim _{\epsilon \downarrow 0} \Theta_{U_{\epsilon}}=\infty .
$$

This computation shows that Theorem 4.2 does not give us any useful information about $\|u(T)-v(T)\|_{L^{1}}$ when the viscosity coefficients tend to zero, as claimed. It also shows that

$$
\lim _{\nu^{\prime} \downarrow 0}\|R(v)\|_{L^{1}\left(L^{1}\right)}=\infty,
$$

which indicates that measuring the residual $R(v)$ in the $L^{1}\left(0, T ; L^{1}\left(\mathbb{R}^{d}\right)\right)$ norm is certainly not a good idea. Next, we show how to overcome this difficulty.

\section{Robustness in the viscosity coefficient}

In this section, we present a new continuous dependence result which, this time, gives meaningful information even when the viscosity coefficients tend to zero. In fact, we can even use it to prove the existence and uniqueness of the entropy solution of

$$
\begin{aligned}
u_{t}+\nabla \cdot \mathbf{f}(u) & =r, & & \text { in } \mathbb{R}^{d} \times(0, T), \\
u(t=0) & =u_{0}, & & \text { on } \mathbb{R}^{d} .
\end{aligned}
$$

Another advantage of this new continuous dependence result is that it also holds for the entropy solution. 
In other words, in this section we find continuous dependence result for the parabolic problems which are automatically inherited by the entropy solution of the hyperbolic problem.

\subsection{Doubling the space variable}

The new estimate is obtained by introducing two changes in the previous approach. The first is to take $v$ to be the solution of

$$
\begin{aligned}
v_{t}+\nabla \cdot \mathbf{f}(v)-\nu^{\prime} \Delta v & =s, & & \text { in } \mathbb{R}^{d} \times(0, T), \\
v(t=0) & =v_{0}, & & \text { on } \mathbb{R}^{d} .
\end{aligned}
$$

Note that the viscosity coefficient $\nu^{\prime}$ is not necessarily equal to the viscosity coefficient $\nu$, as occurred in the previous section.

The second is to measure the difference between the right-hand sides, $r-s$, in a much weaker way. To do that, we introduce the auxiliary function

$$
\varphi_{\epsilon_{x}}(z)=\Pi_{i=1}^{d} \frac{1}{\epsilon_{x}} \omega\left(z_{i} / \epsilon_{x}\right),
$$

where $\omega$ is an even, nonnegative smooth function with support $[-1,1]$ and integral equal to one. We are going to use the following numbers associated with $\omega$ :

$$
\mathrm{c}_{1}=\int_{-1}^{1}|z| \omega(z) \mathrm{d} z \quad \text { and } \quad \mathrm{c}_{2}=\int_{-1}^{1}\left|\omega^{\prime}(z)\right| \mathrm{d} z .
$$

We obtain the following variation of the first continuous dependence result, Theorem 4.2.

Theorem 5.1. Let $u$ be the solution of (4.1) and (4.2) and let $v$ be the solution of (5.3) and (5.4). Let $U$ be given by (4.5). Then,

$$
|u(T)-v(T)|_{U} \leq\left|u_{0}-v_{0}\right|_{U}+\inf _{\epsilon_{x}>0}\left(\mathrm{~A} \epsilon_{x}+\frac{\left(\sqrt{\nu}-\sqrt{\nu^{\prime}}\right)^{2}}{\epsilon_{x}} \mathrm{~B}+\Lambda_{U}^{\epsilon_{x}}(v)\right),
$$

where

$$
\Lambda_{U}^{\epsilon_{x}}(v)=\int_{0}^{T} \int_{\mathbb{R}^{d}} \sup _{c \in \mathbb{R}} E_{U}^{\epsilon_{x}}(c, v ; x, t) \mathrm{d} x \mathrm{~d} t
$$

and

$$
E_{U}^{\epsilon_{x}}(c, v ; x, t)=\int_{\mathbb{R}^{d}} \varphi_{\epsilon_{x}}(x-y) U^{\prime}(c-v(y, t))(r(x, t)-s(y, t)) \mathrm{d} y .
$$

Moreover,

$$
\begin{aligned}
& \mathrm{A}=\mathrm{c}_{1}\left(\min \left\{\|u(T)\|_{T V},\|v(T)\|_{T V}\right\}+\min \left\{\left\|u_{0}\right\|_{T V},\left\|v_{0}\right\|_{T V}\right\}\right), \\
& \mathrm{B}=\mathrm{c}_{2} \min \left\{\|u\|_{L^{1}(T V)},\|v\|_{L^{1}(T V)}\right\} .
\end{aligned}
$$


This result extends the first continuous dependence result, Theorem 4.2, in two ways. First, it renders explicit the influence of the difference of viscosity coefficients. The second is that it measures the difference of right-hand sides in a weaker way; indeed, note that, in the expression of $E_{U}^{\epsilon_{x}}(c, v ; x, t)$, the functions $r=r(x, t)$ and $s=s(y, t)$ depend on different space variables. This doubling of the space variable is a powerful technique that renders this result possible: see the proof in the Appendix. As a consequence, the estimate does not break down when the viscosity coefficients $\nu$ and $\nu^{\prime}$ tend to zero.

\subsection{Letting the viscosity tend to zero: the entropy solution}

We illustrate this phenomenon by showing how to use Theorem 5.1 to establish the existence and uniqueness of the entropy solution. Then we show that Theorem 5.1 also holds when $u$ is taken to be the entropy solution.

Proposition 5.2. Let $u_{i}$ be the solution of (4.1) and (4.2) with $\nu=\nu_{i}$, $i=1,2$. Then

$$
\left\|u_{1}(T)-u_{2}(T)\right\|_{L^{1}} \leq \mathrm{D} \sqrt{24 \mathrm{c}_{1} \mathrm{c}_{2} T}\left|\sqrt{\nu_{1}}-\sqrt{\nu_{2}}\right|,
$$

where $\mathrm{D}=\left\|u_{0}\right\|_{T V}+\|r\|_{L^{1}(T V)}$.

Proof. Since, by (ii) of Proposition 4.3,

$$
\max \left\{\left\|u_{1}\right\|_{L^{\infty}(T V)},\left\|u_{2}\right\|_{L^{\infty}(T V)}\right\} \leq \mathrm{D},
$$

Theorem 5.1 reads

$$
\left\|u_{1}(T)-u_{2}(T)\right\|_{U} \leq \inf _{\epsilon_{x}>0}\left(2 c_{1} \mathrm{D} \epsilon_{x}+\frac{\mathrm{c}_{2}}{\epsilon_{x}}\left(\sqrt{\nu_{1}}-\sqrt{\nu_{2}}\right)^{2} T \mathrm{D}+\Lambda_{U}^{\epsilon_{x}}(v)\right),
$$

and since

$$
\Lambda_{U}^{\epsilon_{x}}(v) \leq \mathrm{c}_{1} \epsilon_{x}\|r\|_{L^{1}(T V)} \leq \mathrm{c}_{1} \epsilon_{x} \mathrm{D}
$$

we have

$$
\left\|u_{1}(T)-u_{2}(T)\right\|_{L^{1}} \leq \inf _{\epsilon_{x}>0}\left(3 c_{1} \epsilon_{x}+c_{2} \frac{\left(\sqrt{\nu_{1}}-\sqrt{\nu_{2}}\right)^{2}}{\epsilon_{x}} T\right) \mathrm{D} .
$$

The result follows by minimizing with respect to the parameter $\epsilon_{x}$. This completes the proof.

The above result states that the sequence $\left\{u_{\nu}\right\}_{\nu>0}$ of exact solutions of the parabolic initial value problem (4.1) and (4.2) is a Cauchy sequence in $\mathcal{C}^{0}\left(0, T ; L^{1}\left(\mathbb{R}^{d}\right)\right)$. As a consequence, it converges to a unique limit $u$ that belongs to $\mathcal{C}^{0}\left(0, T ; L^{1}\left(\mathbb{R}^{d}\right)\right)$, which is in fact merely the entropy solution. Moreover, we immediately have that

$$
\left\|u_{\nu}(T)-u(T)\right\|_{L^{1}} \leq \mathrm{D} \sqrt{24 \mathrm{c}_{1} \mathrm{c}_{2} T} \sqrt{\nu}
$$


Now, we only have to invoke Theorem 5.1 and Proposition 5.2 to obtain the following result.

Theorem 5.3. Theorem 5.1 holds when $u$ is taken to be the entropy of (5.1) and (5.2).

This proves the claim that the entropy solution inherits the continuous dependence results that hold for the solutions of the parabolic problem. A direct consequence of this result is that Propositions 4.3, 4.4 and 4.5 also hold for entropy solutions.

For simplicity, from now on, we are going to assume that

$$
\begin{aligned}
u_{0} & \in L^{\infty}\left(\mathbb{R}^{d}\right) \times B V\left(\mathbb{R}^{d}\right), \\
r & \in L^{\infty}\left(\mathbb{R}^{d} \times(0, T)\right) \cap L^{1}\left(0, T ; B V\left(\mathbb{R}^{d}\right)\right), \\
r_{t} & \in L^{1}\left(0, T ; L^{1}\left(\mathbb{R}^{d}\right)\right), \\
\mathbf{f} & \in \mathcal{C}^{1},
\end{aligned}
$$

where

$$
B V\left(\mathbb{R}^{d}\right)=\left\{v \in L^{1}\left(\mathbb{R}^{d}\right):|v|_{T V}<\infty\right\} .
$$

Note that the above results imply that, in this case, the entropy solution $u$ belongs to the space

$$
\mathcal{C}^{0}\left(0, T ; L^{1}\left(\mathbb{R}^{d}\right)\right) \cap L^{\infty}\left(0, T ; L^{\infty}\left(\mathbb{R}^{d}\right) \cap B V\left(\mathbb{R}^{d}\right)\right),
$$

and that $u_{t}$ belongs to

$$
L^{\infty}\left(0, T ; L^{1}\left(\mathbb{R}^{d}\right)\right) .
$$

Note also that, in the above theorem, $v$ is supposed to be the solution of a parabolic equation instead of a function that could have the same regularity the entropy solution has. In the next section, we show how to overcome this deficiency.

\section{Approximation theory for entropy solutions}

In this section, we present two extensions of Theorem 5.3. In the first, the function $v$ is taken to be in the space of uniformly Lipschitz functions from $[0, T]$ to $L^{1}\left(\mathbb{R}^{d}\right)$. This estimate, however, depends explicitly on the modulus of continuity in time of both $u$ and $v$. In the second extension, we partially remove this constraint and obtain an estimate that is completely independent of $u$, but valid for $v$ as before, or dependent on the modulus of continuity (in time) of $u$, but valid for functions $v$ that only need to be continuous from the left, as functions from $[0, T]$ to $L^{1}\left(\mathbb{R}^{d}\right)$.

The results discussed in this section constitute the main tools for studying approximations to entropy solutions of scalar hyperbolic conservation laws. 


\subsection{Doubling the time variable}

To remove the requirement that the function $v$ be the solution of (4.3) and (4.4) in Theorem 5.1 is quite simple. We only have to replace $s$ by

$$
v_{t}+\nabla_{y} \cdot(v)-\nu^{\prime} \Delta_{y} v
$$

in the expression for $E_{U}^{\epsilon_{x}}$. Moreover, to further reduce the regularity of $v$, we can let $\nu^{\prime}$ tend to zero, in which case we get that

$$
\begin{aligned}
E_{U}^{\epsilon_{x}}(c, v ; x, t)= & \int_{\mathbb{R}^{d}} \varphi_{\epsilon_{x}}(x-y) U^{\prime}(c-v)\left(r-v_{t}-\nabla_{y} \cdot \mathbf{f}(v)\right) \mathrm{d} y \\
= & \int_{\mathbb{R}^{d}} \varphi_{\epsilon_{x}}(x-y)\left(U^{\prime}(c-v)\left(r-v_{t}\right)+\nabla_{y} \cdot \mathbf{G}(c, v)\right) \mathrm{d} y \\
= & \int_{\mathbb{R}^{d}} \varphi_{\epsilon_{x}}(x-y) U^{\prime}(c-v)\left(r-v_{t}\right) \mathrm{d} y \\
& -\int_{\mathbb{R}^{d}} \nabla_{y} \varphi_{\epsilon_{x}}(x-y) \cdot \mathbf{G}(c, v) \mathrm{d} y,
\end{aligned}
$$

where $v=v(y, t)$ and $r=r(x, t)$. We immediately see that, as a function of $y$, the function $v$ can now be discontinuous. This could be achieved because, having doubled the space variable, we were able to integrate by parts. Thus, to be able to do a similar manoeuvre with the time derivative, we only have to double the time variable.

\subsection{The first estimate}

In what follows, we use the auxiliary functions

$$
\varphi_{\epsilon_{t}}(z)=\frac{1}{\epsilon_{t}} \eta\left(z / \epsilon_{t}\right) \quad \text { and } \quad \Phi_{\epsilon_{t}}(t)=2 \int_{0}^{t} \varphi_{\epsilon_{t}}(z) \mathrm{d} z
$$

where $\eta$ is an even, nonnegative smooth function with support $[-1,1]$ and integral equal to one. We have the following extension of Theorem 5.1.

Theorem 6.1. Let $u$ be the entropy solution of (5.1) and (5.2) and let $v$ be any bounded function such that

$$
v \in L^{\infty}\left(0, T ; B V\left(\mathbb{R}^{d}\right)\right) \quad \text { and } \quad v_{t} \in L^{\infty}\left(0, T ; L^{1}\left(\mathbb{R}^{d}\right)\right) .
$$

Let $U$ be given by (4.5). Then,

$$
|u(T)-v(T)|_{U} \leq\left|u_{0}-v_{0}\right|_{U}+\inf _{\epsilon_{x}, \epsilon_{t}>0}\left(\mathrm{~A} \epsilon_{x}+\mathrm{C} \epsilon_{t}+\Lambda_{U}^{\epsilon_{x}, \epsilon_{t}}(v)\right),
$$

where

$$
\Lambda_{U}^{\epsilon_{x}, \epsilon_{t}}(v)=\frac{1}{\Phi_{\epsilon_{t}}(T)} \int_{0}^{T} \int_{\mathbb{R}^{d}} \sup _{c \in \mathbb{R}} E_{U}^{\epsilon_{x}, \epsilon_{t}}(v, c ; x, t) \mathrm{d} x \mathrm{~d} t
$$


and

$$
\begin{gathered}
E_{U}^{\epsilon_{x}, \epsilon_{t}}(v, c ; x, t)=\left.\int_{\mathbb{R}^{d}} \varphi_{\epsilon_{t}, \epsilon_{x}} U(c-v) \mathrm{d} y\right|_{\tau=0} ^{\tau=T}-\int_{0}^{T} \int_{\mathbb{R}^{d}} U(c-v)\left(\varphi_{\epsilon_{t}, \epsilon_{x}}\right)_{t} \mathrm{~d} y \mathrm{~d} \tau \\
-\int_{0}^{T} \int_{\mathbb{R}^{d}}\left(\mathbf{G}(c, v) \cdot \nabla_{y} \varphi_{\epsilon_{t}, \epsilon_{x}}-U^{\prime}(c-v) r \varphi_{\epsilon_{t}, \epsilon_{x}}\right) \mathrm{d} y \mathrm{~d} \tau
\end{gathered}
$$

where $\varphi_{\epsilon_{t}, \epsilon_{x}}=\varphi_{\epsilon_{t}}(t-\tau) \varphi_{\epsilon_{x}}(x-y), r=r(x, t), v=v(y, \tau)$, and

Moreover,

$$
\mathbf{G}(c, v)=\int_{v}^{c} U^{\prime}(c-w) \mathbf{f}^{\prime}(w) \mathrm{d} w .
$$

$$
\begin{aligned}
& \mathrm{A}=\mathrm{c}_{1}\left(\min \left\{\|u(T)\|_{T V},\|v(T)\|_{T V}\right\}+\min \left\{\left\|u_{0}\right\|_{T V},\left\|v_{0}\right\|_{T V}\right\}\right), \\
& \mathrm{C}=\left\|u_{t}\right\|_{L^{\infty}\left(L^{1}\right)}+\left\|v_{t}\right\|_{L^{\infty}\left(L^{1}\right)} .
\end{aligned}
$$

See the Appendix for a proof.

Note that this result allows us to compare the entropy solution $u$ with functions $v$ with much lower regularity than required in Theorem 5.1. Accordingly, an even weaker measure of the residual of $v, \Lambda_{U}^{\epsilon_{x}, \epsilon_{t}}(v)$, is used.

\subsection{The second estimate}

In the result we state next, we take the auxiliary function $\eta$ to be the characteristic function of the interval $[-1,1]$. Let us recall that $\varphi_{\epsilon_{t}}(z)=\frac{1}{\epsilon_{t}} \eta\left(z / \epsilon_{t}\right)$. We have the following variation of Theorem 6.1.

Theorem 6.2. Let $u$ be the entropy solution of (5.1) and (5.2) and let $v$ be any bounded measurable function, continuous from the left as a function from $[0, T]$ into $L^{1}\left(\mathbb{R}^{d}\right)$. Let $U$ be given by (4.5). Then,

$$
|u(T)-v(T)|_{U} \leq 2\left|u_{0}-v_{0}\right|_{U}+4 \inf _{\epsilon_{x}, \epsilon_{t}>0}\left(\mathrm{~A} \epsilon_{x}+\mathrm{C} \epsilon_{t}+\Lambda_{U}^{\epsilon_{x}, \epsilon_{t}}\right),
$$

where $\Lambda_{U}^{\epsilon_{x}, \epsilon_{t}}$ is defined in Theorem 6.1 and

$$
\begin{aligned}
& \mathrm{A}=\mathrm{c}_{1}\left(\min \left\{\|u(T)\|_{T V},\|v(T)\|_{T V}\right\}+\min \left\{\left\|u_{0}\right\|_{T V},\left\|v_{0}\right\|_{T V}\right\}\right), \\
& \mathrm{C}=2 \min \left\{\left\|u_{t}\right\|_{L^{\infty}\left(L^{1}\right)},\left\|v_{t}\right\|_{L^{\infty}\left(L^{1}\right)}\right\} .
\end{aligned}
$$

See the Appendix for a proof.

Note that the constants $\mathrm{A}$ and $\mathrm{C}$ can be bounded by quantities independent of either $u$ or $v$, as desired. We can see that, if the modulus of continuity in time of $v$ is bounded, the estimate is completely independent of the entropy solution $u$. If, on the other hand, we made the constants A and $C$ depend only on $u$, then $v$ does not have to satisfy any continuity restriction. The price we pay for this is innocuous.

In the next two sections, we present applications of the results obtained in this section. First, we obtain a characterization of the entropy solution and then error estimates for the entropy solution and some of its approximations. 


\section{Characterization of the entropy solution}

In this section, we use the a posteriori error estimate of Theorem 6.1 to obtain a characterization of the entropy solution.

However, before treating the general case, we illustrate the technique in the one-dimensional case, $d=1$, when the right-hand side $r$ is equal to zero and the entropy solution is smooth except on a single curve. This result extends the characterization result of weak solutions that are limits of travelling wave solutions of parabolic problems.

\subsection{Piecewise smooth entropy solutions}

Consider the entropy solution of the problem

$$
\begin{aligned}
u_{t}+(f(u))_{x} & =0, & & \text { in } \mathbb{R} \times(0, T), \\
u(t=0) & =u_{0}, & & \text { on } \mathbb{R} .
\end{aligned}
$$

If we assume that $v$ is smooth in two disjoint open sets, namely,

$$
\begin{aligned}
& \Omega^{-}=\{(x, t): x<x(t), t \in(0, T)\}, \\
& \Omega^{+}=\{(x, t): x>x(t), t \in(0, T)\},
\end{aligned}
$$

which are separated by the curve

$$
\Gamma=\{(x(t), t): t \in(0, T)\},
$$

then Theorem 6.1 takes a particularly simple form.

To state it, we need to introduce some notation. We let $\mathbf{n}^{ \pm}$denote the unit normal to $\Gamma$ outward with respect to $\Omega^{ \pm}$and set

$$
v^{ \pm}(x(t), t)=\lim _{h \downarrow 0} v\left((x(t), t)-h \mathbf{n}^{ \pm}\right) .
$$

Finally, we denote the jump of the normal component of the vector $\mathbf{q}(v)$ across the discontinuity curve $\Gamma$ by

$$
\llbracket \mathbf{q}(v) \cdot \mathbf{n} \rrbracket=\mathbf{q}\left(v^{+}\right) \cdot \mathbf{n}^{+}+\mathbf{q}\left(v^{-}\right) \cdot \mathbf{n}^{-} .
$$

We can now state the result.

Corollary 7.1. Let $u$ be the entropy solution of (7.1) and (7.2). Then

$$
\|u(T)-v(T)\|_{L^{1}} \leq\left\|u(0)-v_{0}\right\|_{L^{1}}+\|R(v)\|_{L^{1}(\mathbb{R} \times(0, T) \backslash \Gamma)}+\|\mathcal{R}(v)\|_{L^{1}(\Gamma)},
$$

where

$$
R(v)=v_{t}+(f(v))_{x}
$$

and

$$
\mathcal{R}(v)=\max \left\{0, \sup _{c \in \mathbb{R}}-\llbracket(G(c, v), U(c-v)) \cdot \mathbf{n} \rrbracket\right\} .
$$

In the above expression, $U(e)=|e|$ and $G(u, c)=(f(u)-f(c)) U^{\prime}(u-c)$. 
See the Appendix for a proof.

A direct consequence of this result is the following characterization of the entropy solution. It is obtained by simply realizing that $v=u$ if each of the three terms of the upper bound for $\|u(T)-v(T)\|_{L^{1}}$ is equal to zero.

Proposition 7.2. Assume that there is a function $v$, smooth on the set $\Omega^{-} \cup \Omega^{+}$such that:

(i) $v(t=0)=u_{0}$;

(ii) $v_{t}+(f(v))_{x}=0$ in $\Omega^{-} \cup \Omega^{+}$;

(iii) $\frac{\mathrm{d} x}{\mathrm{~d} t}=\frac{f\left(v^{+}\right)-f\left(v^{-}\right)}{v^{+}-v^{-}}$on $\Gamma$;

(iv) for each $t \in(0, T)$, the graph of $f$ on the interval $\left(v^{-}, v^{+}\right)$(resp., $\left.\left(v^{+}, v^{-}\right)\right)$does not lie below (resp., above) the straight line joining the points $\left(v^{ \pm}, f\left(v^{ \pm}\right)\right)$.

Then $v$ is the entropy solution (7.1) and (7.2).

See the Appendix for a proof.

This result shows that the entropy solution coincides with the strong solution when it is smooth; this is equivalent to requiring that the residual $R(v)$ be zero on the open set $\Omega^{-} \cup \Omega^{+}$. It also shows that if the entropy solution has a curve of discontinuity $\Gamma$, conditions (iii) and (iv) characterize the discontinuity jumps as well as the curve $\Gamma$ itself. In fact, these two properties are equivalent to the condition that the expression $\mathcal{R}(v)$ be identically zero on the discontinuity curve $\Gamma$.

Finally, note that, if the entropy solution is a piecewise constant function, that is, if

$$
u(x, t)= \begin{cases}u^{-}, & x<x(t), \\ u^{+}, & x>x(t),\end{cases}
$$

condition (iii) states that $\Gamma$ is a straight line, and that condition (iv) generalizes the graph condition (2.6) on the discontinuities of entropy solutions that are limits of travelling wave solutions of the parabolic problem.

\subsection{The entropy solution}

The characterizations of the previous subsection rest on the following characterization of the entropy solution in terms of inequality (7.3). This result is in fact a direct consequence of the continuous dependence results obtained for the parabolic solutions.

Theorem 7.3. Assume that the smoothness conditions (5.5), (5.6), (5.7) and (5.8) are satisfied. Then the entropy solution of (5.1) and (5.2) is 
the only measurable bounded function continuous form the left as a function form $[0, T]$ into $L^{1}\left(\mathbb{R}^{d}\right)$ such that, for all nonnegative functions $\varphi$ in $\mathcal{C}_{0}^{\infty}\left(\mathbb{R}^{d} \times[0, T]\right)$,

$$
\mathrm{E}\left(u_{0}, c ; v, \varphi\right) \leq 0, \quad \forall c \in \mathbb{R}
$$

where

$$
\begin{aligned}
\mathrm{E}\left(v_{0}, c ; v, \varphi\right)= & \int_{\mathbb{R}^{d}} \varphi(T) U(c-v(T)) \mathrm{d} y-\int_{\mathbb{R}^{d}} \varphi U\left(c-v_{0}\right) \mathrm{d} y \\
& -\int_{0}^{T} \int_{\mathbb{R}^{d}}\left(U(c-v) \varphi_{t}+\mathbf{G}(c, v) \cdot \nabla_{y} \varphi-U^{\prime}(c-v) r \varphi\right) \mathrm{d} y \mathrm{~d} \tau
\end{aligned}
$$

where

$$
U(e)=|e| \quad \text { and } \quad \mathbf{G}(c, v)=\int_{v}^{c} U^{\prime}(c-w) \mathbf{f}^{\prime}(w) \mathrm{d} w .
$$

For a proof, see the Appendix. Here, let us simply point out that, for $r=0$, the link between the continuous dependence Theorems 6.1 and 6.2 is established by the following simple equality:

$$
E_{U}^{\epsilon_{x}, \epsilon_{t}}(v, c ; x, t)=\mathrm{E}\left(u_{0}, c ; v, \varphi_{\epsilon_{x}, \epsilon_{t}}\right) .
$$

We can now show that the entropy solution is also the physically relevant solution with respect to the following model:

$$
\left(u_{\omega}\right)_{t}+\nabla \cdot \mathbf{f}\left(u_{\omega}\right)-\nabla \cdot \mathbb{L}\left(\omega ; u_{\omega}, \nabla u_{\omega}\right)=0,
$$

where $\mathbb{L}(0 ; \cdot, \cdot)=0$, provided

$$
\mathbb{L}(\cdot ; \cdot, p) \cdot p \geq 0 \quad \forall p \in \mathbb{R}^{d},
$$

and

$$
\lim _{\omega \downarrow 0} \int_{0}^{T} \int_{\mathbb{R}^{d}}\left|\mathbb{L}\left(\omega ; u_{\omega}, \nabla u_{\omega}\right)\right| \mathrm{d} x \mathrm{~d} t=0 .
$$

Let us show that this is indeed true for the case $r=0$. Thus, for any nonnegative test function $\varphi$, we have

$$
\begin{aligned}
\mathrm{E}\left(u_{0}, c ; u_{\omega}, \varphi\right) & =-\int_{0}^{T} \int_{\mathbb{R}^{d}} U^{\prime}\left(c-u_{\omega}\right)\left(\left(u_{\omega}\right)_{t}+\nabla \cdot \mathbf{f}\left(u_{\omega}\right)\right) \varphi \mathrm{d} x \mathrm{~d} t \\
& =\mathrm{E}_{\text {small }}+\mathrm{E}_{\text {diss }},
\end{aligned}
$$

where

$$
\begin{aligned}
\mathrm{E}_{\mathrm{diss}} & =\int_{0}^{T} \int_{\mathbb{R}^{d}} U^{\prime}\left(c-u_{\omega}\right) \mathbb{L}\left(\omega ; u_{\omega}, \nabla u_{\omega}\right) \cdot \nabla \varphi \mathrm{d} x \mathrm{~d} t \\
\mathrm{E}_{\text {small }} & =-\int_{0}^{T} \int_{\mathbb{R}^{d}} U^{\prime \prime}\left(c-u_{\omega}\right) \mathbb{L}\left(\omega ; u_{\omega}, \nabla u_{\omega}\right) \cdot \nabla u_{\omega} \varphi \mathrm{d} x \mathrm{~d} t .
\end{aligned}
$$


Since $\varphi$ is nonnegative, $U$ is convex, and $\mathbb{L}$ satisfies the positivity condition (7.4), we have that

$$
\mathrm{E}_{\text {diss }} \leq 0 \text {. }
$$

Moreover, it is easy to see that, by the convergence condition (7.5),

$$
\mathrm{E}_{\text {small }} \leq\|\nabla \varphi\|_{L^{\infty}\left(L^{\infty}\right)} \int_{0}^{T} \int_{\mathbb{R}^{d}}\left|\mathbb{L}\left(\omega ; u_{\omega}, \nabla u_{\omega}\right)\right| \mathrm{d} x \mathrm{~d} t \rightarrow 0
$$

as $\omega \downarrow 0$. Of course, we assumed that $u_{\omega}(t=0)=u_{0}$. This extends a similar result obtained in Section 3.1 for travelling wave solutions.

\subsection{The entropy inequality}

The inequality (7.3) is called entropy inequality as it is reminiscent of a similar situation for compressible fluid flow. Indeed, the conservation of the entropy $\rho s$ given by the compressible Navier-Stokes equations reads

$$
(\rho s)_{t}+\left(\rho s v_{j}\right)_{, j}=\psi
$$

where $\rho s \mathbf{v}$ is the entropy flux and

$$
\psi=\frac{\lambda}{T}\left(v_{i, i}\right)^{2}+\frac{\mu}{2 \lambda}\left(v_{i, j}+v_{j, i}-\frac{2}{3} v_{k, k} \delta_{i j}\right)^{2}+\frac{1}{T}\left(\kappa T_{, i}\right)_{, i} .
$$

If we multiply the conservation law by a nonnegative $\varphi$ in $\mathcal{C}_{0}^{\infty}\left(\mathbb{R}^{d} \times[0, T]\right)$ and integrate, we get

$$
\begin{aligned}
\mathbb{E}(\varphi)= & \int_{\mathbb{R}^{d}} \varphi(T)(\rho s)(T) \mathrm{d} y-\int_{\mathbb{R}^{d}} \varphi(0)(\rho s)(0) \mathrm{d} y \\
& -\int_{0}^{T} \int_{\mathbb{R}^{d}}\left((\rho s) \varphi_{t}+\rho s \mathbf{v} \cdot \nabla_{y} \varphi\right) \mathrm{d} y \mathrm{~d} \tau \\
= & \int_{0}^{T} \int_{\mathbb{R}^{d}} \psi \varphi \mathrm{d} y \mathrm{~d} \tau
\end{aligned}
$$

where $\psi$ is given by (3.1). This implies that

$$
\mathbb{E}(\varphi)=\mathbb{E}_{\text {small }}+\mathbb{E}_{\text {diss }}
$$

where

$$
\begin{aligned}
\mathbb{E}_{\text {small }} & =-\kappa \int_{0}^{T} \int_{\mathbb{R}^{d}} \frac{1}{T} \nabla T \cdot \nabla \varphi \mathrm{d} y \mathrm{~d} \tau, \\
\mathbb{E}_{\text {diss }} & =\int_{0}^{T} \int_{\mathbb{R}^{d}} \Psi \varphi \mathrm{d} y \mathrm{~d} \tau,
\end{aligned}
$$

and $\Psi$ is given by (3.2). By the second law of thermodynamics, $\Psi \geq 0$, and so

$$
\mathbb{E}_{\text {diss }} \geq 0 \text {. }
$$


Moreover, if

$$
\mathbb{E}_{\text {small }} \rightarrow 0 \text {, }
$$

as $\kappa \downarrow 0$, we get the so-called entropy inequality

$$
\mathbb{E}(\varphi) \geq 0 .
$$

Because of this, and since the function $U(c-u)$ has a role analogous to that of the entropy $\rho s$, and $G(c, u)$ a role analogous to that of the entropy flux $\rho s \mathbf{v}$, they are usually referred to in the same manner.

\section{Error estimates for the Engquist-Osher scheme}

In this section, we apply the a posteriori error estimates of Section 6 to obtain an estimate of the distance between the entropy solution of the simple Cauchy problem

$$
\begin{aligned}
u_{t}+\nabla \cdot \mathbf{f}(u) & =0, & & \text { in } \mathbb{R} \times(0, T), \\
u(t=0) & =u_{0}, & & \text { on } \mathbb{R},
\end{aligned}
$$

and the approximate solution, $u_{h}$, determined by a model monotone numerical scheme, the Engquist-Osher scheme.

To define the scheme, we discretize the time interval $(0, T)$ in intervals $J^{n}=\left(t^{n}, t^{n+1}\right)$ and set $\Delta^{n}=t^{n+1}-t^{n}$ and $t^{N}=T$. We also discretize $\mathbb{R}$ in intervals $I_{j}=\left(x_{j-1 / 2}, x_{j+1 / 2}\right)$ and set $\Delta_{j}=x_{j+1 / 2}-x_{j-1 / 2}$. The approximate solution $u_{h}$ is continuous from the left in time and is equal to the value $u_{i}^{n}$ on the rectangle $I_{i} \times J^{n}$.

These values are determined in the following way. For $n=0, \ldots, N-1$,

$$
\begin{aligned}
\frac{u_{j}^{n+1}-u_{j}^{n}}{\Delta^{n}}+\frac{\widehat{f}_{j+1 / 2}^{n}-\widehat{f}_{j-1 / 2}^{n}}{\Delta_{j}} & =0, \quad \forall j \in \mathbb{Z}, \\
u_{j}^{0} & =\frac{1}{\Delta_{j}} \int_{I_{j}} u_{0}(x) \mathrm{d} x, \quad \forall j \in \mathbb{Z},
\end{aligned}
$$

and the so-called Engquist-Osher numerical flux $\widehat{f}_{j+1 / 2}^{n}=\widehat{f}\left(u_{i}^{n}, u_{i+1}^{n}\right)$ is given by

$$
\widehat{f}(a, b)=f^{+}(a)+f^{-}(b),
$$

where

$$
f^{+}(a)=\int_{d}^{a} \max \left\{0, f^{\prime}(s)\right\} \mathrm{d} s, \quad f^{-}(b)=-\int_{d}^{b} \max \left\{0,-f^{\prime}(s)\right\} \mathrm{d} s,
$$

and $d$ is an arbitrary, but fixed, real value.

We could use the a posteriori error estimate of Theorem 6.1 with $v=\tilde{u}_{h}$ if we take $\tilde{u}_{h}$ to be the continuous function equal to the value $u_{i}^{n}$ at the 
point $\left(x_{i}, t^{n}\right)$. We can also apply Theorem 6.2 with $v=u_{h}$ without any modification. Of course, we can always insert all the information about the approximate solution $u_{h}$ and then simplify the corresponding upper bound as much as possible. Next, we consider the result of such operations.

To state it, we introduce the rate of entropy dissipation (RED) of the numerical scheme. It is a piecewise constant function whose value for $(x, t) \in$ $I_{n} \times J^{n}$ is

$$
\operatorname{RED}(x, t)=\sup _{c \in \mathbb{R}}\left(\frac{U\left(c-u_{j}^{n+1}\right)-U\left(c-u_{j}^{n}\right)}{\Delta^{n}}+\frac{\widehat{G}_{j+1 / 2}^{n}(c)-\widehat{G}_{j-1 / 2}^{n}(c)}{\Delta_{j}}\right),
$$

where the numerical entropy flux $\widehat{G}_{j+1 / 2}^{n}(c)=G^{+}\left(c, u_{j}^{n}\right)+G^{-}\left(c, u_{j+1}^{n}\right)$ is given by

$$
G^{ \pm}(c, v)=\int_{v}^{c} U^{\prime}(c-s)\left(f^{ \pm}\right)^{\prime}(s) \mathrm{d} s .
$$

Theorem 8.1. Let $u$ be the entropy solution of (8.1) and (8.2) and let $u_{h}$ be the approximate solution of the Engquist-Osher scheme (8.3) and (8.4). Let $U$ be given by (4.5). Then,

$$
\left|u(T)-u_{h}(T)\right|_{U} \leq \Phi\left(u_{h}\right),
$$

where

$$
\Phi\left(u_{h}\right)=2\left|u_{0}-u_{h}(0)\right|_{U}+C\left|u_{0}\right|_{T V} \Theta_{0}^{1 / 2}+\int_{0}^{T} \int_{\mathbb{R}} \operatorname{RED~d} x \mathrm{~d} t
$$

where

$$
\Theta_{0}=\sum_{n=0}^{N-1} \sum_{j \in \mathbb{Z}}\left(\left|\widehat{f}_{j+1 / 2}^{n}-f\left(u_{j}^{n}\right)\right|+\left|\widehat{f}_{j-1 / 2}^{n}-f\left(u_{j}^{n}\right)\right|\right) \Delta_{j} \Delta^{n} .
$$

Proof. Then, by Theorem 6.2 we have

$$
\left|u(T)-u_{h}(T)\right|_{U} \leq 2\left|u_{0}-u_{h}(0)\right|_{U}+2 \inf _{\epsilon_{x}, \epsilon_{t}>0}\left(\mathrm{~A} \epsilon_{x}+\mathrm{C} \epsilon_{t}+\Lambda_{U}^{\epsilon_{x}, \epsilon_{t}}\right),
$$

where

$$
\Lambda_{U}^{\epsilon_{x}, \epsilon_{t}}=\frac{1}{\Phi_{\epsilon_{t}}(T)} \int_{0}^{T} \int_{\mathbb{R}^{d}} \sup _{c \in \mathbb{R}} E_{U}^{\epsilon_{x}, \epsilon_{t}}(v, c ; x, t) \mathrm{d} x \mathrm{~d} t .
$$

A simple computation gives

$$
\Lambda_{U}^{\epsilon_{x}, \epsilon_{t}}=\Lambda_{\text {small }}+\Lambda_{\text {diss }}
$$

where

$$
\Lambda_{\text {small }}=\left(\frac{1}{\epsilon_{t}}+\frac{1}{\epsilon_{x}}\right) \Theta_{0}, \quad \Lambda_{\text {diss }}=\int_{0}^{T} \int_{\mathbb{R}} \operatorname{RED~d} x \mathrm{~d} t .
$$

The result follows after minimizing on $\epsilon_{t}$ and $\epsilon_{x}$. This completes the proof. 
If we base an adaptive algorithm on the above estimate, it is important to know if given an arbitrary tolerance TOL, it is possible to find a mesh such that the quantity $\Phi\left(u_{h}\right)$ can actually be made smaller than TOL. It is not difficult to see that, in fact, this can always be done.

Indeed, since it is possible to show that

$$
\begin{aligned}
& \left|u_{0}-u_{h}(0)\right|_{U} \leq\left|u_{0}\right|_{T V} h, \quad \operatorname{RED}_{j}^{n} \leq 0, \\
& \sum_{j \in \mathbb{Z}}\left|f\left(u_{j+1}^{n}\right)-f\left(u_{j}^{n}\right)\right| \leq \sup _{w \in R g\left(u_{0}\right)}\left|f^{\prime}(w)\right|\left|u_{0}\right|_{T V},
\end{aligned}
$$

provided that

$$
\sup _{w \in R g\left(u_{0}\right)}\left|f^{\prime}(w)\right| \sup _{j \in \mathbb{Z}} \frac{\Delta^{n}}{\Delta_{j}} \leq 1
$$

where $R g\left(u_{0}\right)$ is the convex hull of the range of $u_{0}$, we immediately see that we get the a priori estimate

$$
\Phi\left(u_{h}\right) \leq C(\Delta x)^{1 / 2},
$$

for some constant $C$ independent of $\Delta x=\sup _{j \in \mathbb{Z}} \Delta_{j}$.

This concludes the presentation of the material we wanted to discuss in this paper. It has not followed the historical development of the topic, but rather the short introduction to the subject for graduate students given in Cockburn (1999). No references were provided, in order to focus on the ideas themselves. Next, we compensate for this omission and present a brief overview of the historical development of the topic under consideration.

\section{The historical evolution of some ideas}

\subsection{A priori error estimates I}

The definition of the entropy solution (in terms of the entropy inequality) was first introduced in the seminal paper by Kružkov (1970). Therein, Kružkov proved its existence, uniqueness and stability with respect to initial data. He proved the existence of the entropy solution by using the vanishing viscosity method. First, he obtained estimates of moduli of continuity of the solutions, $u_{\nu}$, of the parabolic problem which are independent of the viscosity coefficient, $\nu$. Then, he used a compactness argument to show that, when $\nu$ tends to zero, the functions $u_{\nu}$ converge to a limit that satisfies the entropy inequality. He obtained uniqueness and stability by using the entropy inequality and the doubling of variables technique (which was also introduced in this paper). For our simple model scalar conservation law, the continuous dependence result

$$
\left\|u_{1}(T)-u_{2}(T)\right\|_{L^{1}} \leq\left\|u_{0,1}(T)-u_{0,2}(T)\right\|_{L^{1}}
$$


was obtained, where $u_{i}$ is the entropy solution of the Cauchy problem with initial data $u_{0, i}, i=1,2$; see Proposition 4.4 and Theorem 5.3. No other error estimate was obtained.

Error estimates and, in fact, a powerful approximation theory for the entropy solution was developed a few years later by Kuznetsov (1976). The main result is (essentially) what we have labelled as Theorem 6.1. It was applied to obtain the first a priori error estimate between the entropy solution $u$ and the parabolic solution $u_{\nu}$ (when $r=0$ ), namely,

$$
\left\|u-u_{\nu}\right\|_{L^{\infty}\left(L^{1}\right)} \leq C\left|u_{0}\right|_{T V} \sqrt{T \nu}
$$

and the first a priori error estimate between the entropy solution and the approximate solution $u_{h}$ defined by a monotone scheme on uniform Cartesian grids

$$
\left\|u-u_{h}\right\|_{L^{\infty}\left(L^{1}\right)} \leq C\left|u_{0}\right|_{T V} \sqrt{T \Delta x} .
$$

(The rate of convergence of $(\Delta x)^{1 / 2}$ is the best possible rate for monotone schemes when the initial data are functions of bounded variation, as was shown many years later in Şabac (1997).)

Around the same time, the paper by Harten, Hyman and Lax (1976) on monotone schemes appeared, which gave a physical argument indicating that convergence to the entropy solution should always take place. Moreover, it was shown that monotone schemes are necessarily at most first-order accurate, and that second- (and higher)-order schemes might not converge to the entropy solution. To illustrate this, we show in Figure 9.1 the entropy solution of the problem

$$
u_{t}+\left(u^{2} / 2\right)_{x}=0, \quad u(x, t=0)= \begin{cases}1, & x \in(.4, .6), \\ 0, & \text { otherwise }\end{cases}
$$

and the approximation given by a monotone scheme, the Engquist-Osher scheme, and a formally second-order accurate scheme, the Lax-Wendroff method.

A few years later, convergence of monotone schemes (in uniform Cartesian grids) to the entropy solution was proved by Crandall and Majda (1980). A compactness argument was used which could be considered to be the discrete counterpart of the argument used in Kružkov (1970). Apparently, the authors of this result were unaware of the approximation theory introduced in Kuznetsov (1976).

However, this theory has since been used by most researchers working on error estimation for scalar hyperbolic conservation laws. Thus, Sanders (1983) used it to prove that

$$
\left\|u-u_{h}\right\|_{L^{\infty}\left(L^{1}\right)} \leq C\left|u_{0}\right|_{T V} \sqrt{T \Delta x}
$$



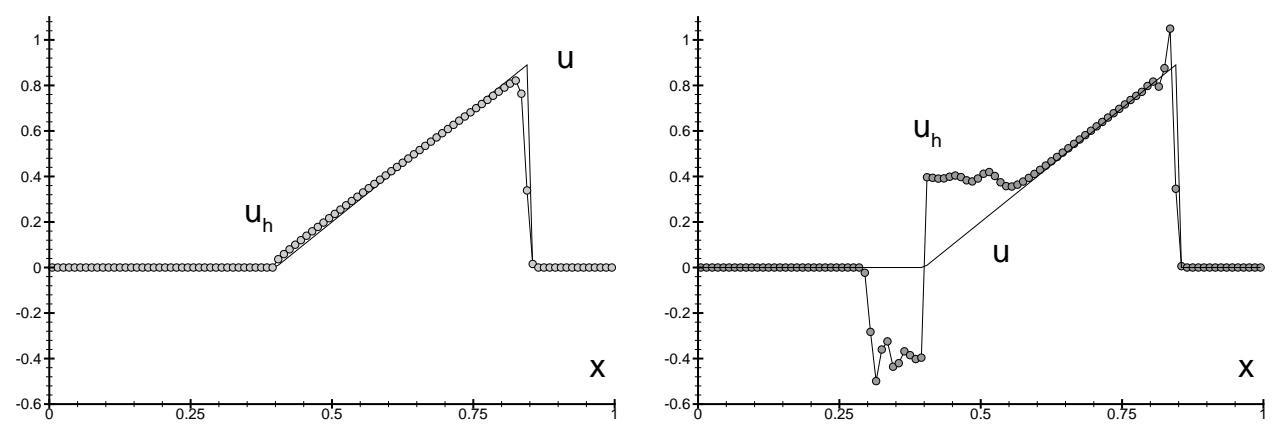

Figure 9.1. The entropy solution, $u$, and its approximation $u_{h}$ at time $T=1 / 2$ : Engquist-Osher scheme (left) and Lax-Wendroff scheme (right).

for $u_{h}$ determined by monotone schemes defined in non-uniform Cartesian grids. His main contribution was to show that the total variation of the approximation $u_{h}$ was uniformly bounded in time. This estimate immediately implies the uniform estimate of the modulus of continuity in time, essential for the application of Theorem 6.1.

Then Lucier (1985a) obtained the bound

$$
\left\|u-u_{h}\right\|_{L^{\infty}\left(L^{1}\right)} \leq\left|u_{0}\right|_{T V}\left(\Delta x+\frac{2}{\sqrt{3}} \frac{\Delta x T^{1 / 2}}{(\Delta t)^{1 / 2}}\right),
$$

for the numerical schemes of Godunov, Glimm and LeVeque in one space dimension. To prove the estimate for LeVeque's method, he had to prove that, if $u_{i}$ is the entropy solution with $\mathbf{f}=\mathbf{f}_{i}, i=1,2$, then

$$
\left\|u_{1}-u_{2}\right\|_{L^{\infty}\left(L^{1}\right)} \leq C T\left|u_{0}\right|_{T V}\left\|\mathbf{f}_{1}^{\prime}-\mathbf{f}_{2}^{\prime}\right\|_{L^{\infty}(I)} ;
$$

see the similar result for the parabolic solutions in Proposition 4.4 and Theorem 5.3.

Later, error estimates of the form

$$
\left\|u-u_{h}\right\|_{L^{\infty}\left(L^{1}\right)} \leq C\left|u_{0}\right|_{T V} \sqrt{T}(\Delta x)^{\delta},
$$

where $\delta \in(0,1 / 2]$, for the so-called quasi-monotone schemes (which include Petrov-Galerkin methods) were obtained in Cockburn $(1989,1990 a, 1990 b)$ for the case of Cartesian grids. The parameter $\delta$ is equal to $1 / 2$ for monotone schemes and controls the spurious production of entropy per cell typical of high-order accurate schemes. The main approximation result in this papers was the modification of the approximation theory introduced in Kuznetsov (1976) to include what we have called the smooth functions $U$, and its application to non-monotone schemes defined in several space dimensions. 


\subsection{A priori error estimates II}

In all the above papers, an estimate of the modulus of continuity in time was obtained, as required by Theorem 6.1. However, to obtain such an estimate is extremely difficult; indeed, there is no known numerical scheme defined in unstructured grids for which this property has been proved. This is true even for the monotone schemes, the simplest schemes for hyperbolic conservation laws.

To obtain a priori error estimates thus became an extremely difficult task, and the main focus in this area shifted to the search for weaker smoothness properties with which convergence, and not error estimates, could be proved. Thus, the theory of convergence of measure-valued functions (DiPerna 1985) was used. (See the monograph by Málek, Nečas, Rokyta and Ružička (1996) on weak and measure-valued solutions for a treatment of the initial boundary-value problem.) This was first done by Szepessy $(1989,1991)$, where the streamline diffusion method with shock-capturing terms was proved to converge to the entropy solution; a refinement of this approach was obtained a few years later by Jaffré, Johnson and Szepessy (1995). This approach was also used by Coquel and LeFloch (1991) to prove convergence of finite difference methods, and by Cockburn, Coquel and LeFloch (1995), Kröner, Noelle and Rokyta (1995) and Nöelle (1995) to prove finite volume methods defined in unstructured triangulations.

Since the main difficulty in using the approximation theory introduced by Kuznetsov (1976) was the estimate of the modulus of continuity in time of the approximate solution (the constant $C$ in Theorem 6.1 ), a switch to Theorem 6.2 would solve the problem since the corresponding $C$ is independent of such a modulus. This was noticed by Cockburn, Coquel and LeFloch (1994), Kröner and Rokyta (1994) and Vila (1994), where it was shown that

$$
\left\|u-u_{h}\right\|_{L^{\infty}\left(L^{1}\right)} \leq C\left|u_{0}\right|_{T V}^{1 / 2}\left\|u_{0}\right\|_{L^{2}}^{1 / 2}(\Delta x)^{1 / 4}
$$

where $u_{h}$ is given by monotone methods defined in general triangulations; see also Nöelle (1996). This approach was further developed in Cockburn and Gremaud (1996a), where a similar estimate was proved for the shockcapturing discontinuous Galerkin method and the estimate

$$
\left\|u-u_{h}\right\|_{L^{\infty}\left(L^{1}\right)} \leq C\left|u_{0}\right|_{T V}^{1 / 2}\left\|u_{0}\right\|_{L^{2}}^{3 / 4}(\Delta x)^{1 / 8}
$$

was shown for the shock-capturing streamline diffusion method. The lack of optimality in the rate of convergence is due to the fact that the total variation of the approximate solution cannot be proved to be uniformly bounded in time. This lack of uniform boundedness of the total variation has a greater impact on the streamline diffusion method, because of its use 
of continuous approximations. In fact, only a weaker estimate of the form

$$
\left(\int_{0}^{T} \int_{\mathbb{R}^{d}}|\nabla u|^{2} \mathrm{~d} x\right)^{1 / 2} \leq\left\|u_{0}\right\|_{L^{2}\left(\mathbb{R}^{d}\right)}(\Delta x)^{-1 / 2}
$$

can be proved. The discrete version of this estimate relies on the continuity of the $L^{2}$ projection into finite-dimensional spaces of discontinuous functions: see Cockburn (1991).

Finally, let us point out that, for each of the above two methods, the approximate solution is a piecewise polynomial of degree $k \geq 0$. The fact that the a priori estimates do not reflect the influence of the polynomial degree is certainly a drawback.

Extensions of these results to the conservation law

$$
u_{t}+\nabla \cdot(\mathbf{v} f(u))=0,
$$

where $\mathbf{v}$ is a divergence-free function, were obtained in Eymard, Gallouët, Ghilani and Herbin (1998), where the estimate (9.1) was proved. Extensions to the conservation law

$$
u_{t}+\nabla \cdot \mathbf{F}(x, t, u)=0
$$

where $\nabla_{x} \cdot \mathbf{F}=0$, were carried out in Chainais-Hillairet (1999). More importantly, the error $\left\|u(T)-u_{h}(T)\right\|_{L^{1}(\Omega)}$ was bounded in terms of the behaviour of $u_{h}$ in the domain of dependence associated with $\Omega$; this constitutes the first local error estimate for this kind of problem. See Chainais-Hillairet and Champier (2001) for extensions to the case in which the right-hand side is not equal to zero.

A formalization of the techniques used in the above-mentioned papers was obtained in Bouchut and Perthame (1998). In particular, the following fundamental fact was identified. If, for $U(e)=|e|$, we have that

$$
U^{\prime}(v-c) R(v) \leq \nabla \cdot H_{c}
$$

then

$$
\|u(T)-v(T)\|_{L^{1}} \leq\left\|u_{0}-v_{0}\right\|_{L^{1}}+C\|H\|_{L^{1}\left(L^{1}\right)}^{1 / 2},
$$

if $\left|H_{c}\right| \leq|H|$ for all $c \in \mathbb{R}$. Let us also mention that, using this technique, the following result was obtained. If $u_{i}$ is the entropy solution with $r=0$, $\mathbf{f}=\mathbf{f}_{i}, i=1,2$, with $\mathbf{f}_{1}(0)=\mathbf{f}_{2}(0)$, then

$$
\left\|u_{1}-u_{2}\right\|_{L^{\infty}\left(L^{1}\right)} \leq C\left(T\left|u_{0}\right|_{L^{1}}\left|u_{0}\right|_{T V} Q\right)^{1 / 2},
$$

where

$$
Q=\sup _{\xi \in \mathbb{R} \backslash\{0\}} \frac{\left|\mathbf{f}_{1}(\xi)-\mathbf{f}_{2}(\xi)\right|}{|\xi|} .
$$

Compare this with the continuous dependence result (iv) of Proposition 4.4. 


\subsection{A priori error estimates III}

Lucier (1986) considered and analysed moving-mesh methods in one space dimension. The methods used approximate solutions $u_{h}$ which were piecewise constant or piecewise linear between points called nodes; these nodes were then evolved in a suitable way. For strictly convex, smooth nonlinearities $f$, and $N$ nodes, it was shown that

$$
\left\|u(T)-u_{h}(T)\right\|_{L^{1}} \leq C N^{-1},
$$

for piecewise constant approximations (Dafermos' method) and

$$
\left\|u(T)-u_{h}(T)\right\|_{L^{1}} \leq C N^{-2},
$$

for piecewise linear approximations. These are remarkable results, not only because the rate of convergence is not the classical rate of order $N^{-1 / 2}$, but because they give information about the approximability of the entropy solution by functions that are piecewise polynomials between nodes. From these results, regularity properties of the exact solution can be deduced.

This idea was developed in the papers by Lucier (1988) and DeVore and Lucier $(1990,1996)$. In particular, it was shown that, if the initial data $u_{0}$ belongs to the Besov space $B_{\sigma}^{\alpha}\left(L^{\sigma}\right)$, where $\sigma=1 /(1+\alpha)$ and $\alpha>0$, then the same is true for the entropy solution $u(\cdot, t)$ for $t>0$. It was also shown that this property does not hold for any Besov space $B_{\sigma}^{\alpha}\left(L^{\sigma}\right)$ with $\alpha>1$. Note that, since $\sigma<1$, the space $L^{\sigma}$ is not a locally convex space.

\subsection{A priori error estimates $I V$}

Note that Theorem 6.2 gives an estimate of the form

$$
\left\|u-u_{h}\right\|_{L^{\infty}\left(L^{1}\right)} \leq \Phi\left(u_{h}\right) .
$$

This means that, in order to obtain an a priori error for the above estimate, we are bound to obtain regularity properties of the approximate solution $u_{h}$, and this is extremely difficult, as we have seen. An ideal way out of this unfortunate predicament would be to be able to interchange the roles of $u$ and $u_{h}$ in Theorem 6.2 , as we would then obtain an estimate of the form

$$
\left\|u_{h}-u\right\|_{L^{\infty}\left(L^{1}\right)} \leq \Phi_{h}(u) .
$$

In this way, the estimate would be totally independent of the regularity properties of $u_{h}$.

The main idea is to realize that, while $\Phi\left(u_{h}\right)$ is a measure of the residual of $u_{h}$, the functional $\Phi_{h}(u)$ should be nothing but a measure of the truncation error of $u$. Thus, instead of basing the approximation theory on the entropy inequality for $u$, it would be enough to base it on the discrete entropy entropy inequality for the approximate solution $u_{h}$. That this can actually be carried out was proved in the papers of Cockburn and Gremaud $(1996 b, 1997)$ and 
Cockburn, Gremaud and Yang (1998), when $u_{h}$ is defined by monotone schemes. In particular, it was proved that

$$
\left\|u-u_{h}\right\|_{L^{\infty}\left(L^{1}\right)} \leq C\left|u_{0}\right|_{T V} \sqrt{T \Delta x}
$$

for the Lax-Friedrichs method (monotone scheme) in a uniform grid of triangles without using any regularity properties of the approximate solution. This result cannot be proved with any other technique.

The application of this approach to other numerical schemes remains to be carried out.

\subsection{A posteriori error estimates and adaptivity}

We thus see that the theory of a priori error estimates for entropy solutions, which should be based on estimates of the truncation error, was slowed down because it was based on Theorems 6.1 and 6.2 , which are based on estimates of the residual instead. For this reason, they are, so to speak, natural a posteriori error estimates. Conversely, the use of these a posteriori error estimates as a stepping stone to obtain a priori error estimates also obscured the fact that they could be used as the basis of adaptive algorithms.

These estimates were for the first time recognized as such in Cockburn and Gremaud (1996a), where a posteriori error estimates for the shock-capturing streamline diffusion and the discontinuous Galerkin methods of arbitrary order were obtained; however, they still remain to be numerically tested. On the other hand, this lack of clarity did not prevent the introduction of the first stable adaptive algorithm for scalar hyperbolic conservation laws in Lucier (1985b). Numerical evidence was shown which indicated that, if an error of size $\epsilon$ is required, a computational complexity of order $\epsilon^{-3}$ was required instead of the complexity of standard monotone schemes of order $\epsilon^{-4}$. See also Lucier and Overbeek (1987).

Later, adaptive algorithms were implemented by Kröner and Ohlberger (2000) for a monotone scheme, and then by Gosse and Makridakis (2000) for monotone second-order finite-difference schemes. The implementation by Kröner and Ohlberger (2000) used a local version of Theorem 6.1 obtained in Chainais-Hillairet (1999).

The only study of the ratio of the upper bound of the error to the error itself, usually called the effectivity index, has been done in Cockburn and Gau (1995) for the Engquist-Osher scheme in one space dimension. There, the a posteriori error estimate of Corollary 7.1 was obtained and then applied to a continuous approximate solution $u_{h}$ obtained by a bilinear interpolation of the values $u_{j}^{n}$. The effectivity index was shown to remain close to one for smooth entropy solutions and for entropy solutions with discontinuities and linear convection. The case of nonlinear convection and entropy solutions with discontinuities was not treated therein; it remains to be studied. 


\subsection{Continuous dependence for parabolic problems}

The technique used in Kuznetsov (1976) to obtain error estimates for the entropy solution was thought to be impossible to extend to the parabolic case. However, this extension was actually carried out by Cockburn and Gripengerg (1999), who obtained continuous dependence of the solution of the scalar degenerate parabolic equation

$$
u_{t}+\nabla \cdot \mathbf{f}(u)-\Delta \varphi(u)=0
$$

on the nonlinearities $\mathbf{f}$ and $\varphi$. The main continuous dependence result for parabolic solutions, Theorem 5.1, which is the basis for the approximation theory for entropy solutions, Theorems 6.1 and 6.2 , was proposed in Cockburn (1999), and is based on the technique introduced in Cockburn and Gripengerg (1999). A simple exposition of this approach can be found in the proof of Lemma A1 in the Appendix.

In particular, Cockburn and Gripengerg (1999) proved that, if $u_{i}$ is the (semi-group) solution of the above equation with $\varphi=\varphi_{i}$, and $u_{i}(t=0)=u_{0}$, for $i=1,2$, then

$$
\left\|u_{1}-u_{2}\right\|_{L^{\infty}\left(L^{1}\right)} \leq C\left\|\sqrt{\varphi_{1}^{\prime}}-\sqrt{\varphi_{2}^{\prime}}\right\|_{L^{\infty}(I)},
$$

where $I$ is the convex hull of the range of $u_{0}$. We note that Benilan and Crandall (1981) studied the dependence with respect to $\varphi$ in the case $\mathbf{f}=\mathbf{0}$, but their results are not written in terms of explicit estimates. This means that a complete approximation theory for entropy solutions of degenerate parabolic equations can be obtained in exactly the same way as for the entropy solution of the scalar hyperbolic conservation law.

On the other hand, an approximation theory for degenerate parabolic equations can also be constructed from the characterization of their entropy solutions obtained in Carrillo (1999) (see also the extension to more general boundary conditions in Mascia, Porretta and Terracina (2002)), just as the approximation theory in Kuznetsov (1976) was obtained from the characterization of the entropy solution for scalar hyperbolic conservation laws given in Kružkov (1970). Using this approach, comparisons between the entropy solution of

$$
\left(u_{1}\right)_{t}+\nabla \cdot\left(\mathbf{v} f\left(u_{1}\right)\right)-\Delta \varphi\left(u_{1}\right)=0
$$

and that of

$$
\left(u_{2}\right)_{t}+\nabla \cdot\left(\mathbf{v} f\left(u_{2}\right)\right)-\Delta\left(\varphi\left(u_{2}\right)+\epsilon u_{2}\right)=0
$$

have recently been obtained. Indeed, Evje and Karlsen (2002) proved that

$$
\left\|u_{1}-u_{2}\right\|_{L^{\infty}\left(L^{1}\right)} \leq C \epsilon^{1 / 2},
$$

and Eymard, Gallouët and Herbin (2002a) proved that

$$
\left\|u_{1}-u_{2}\right\|_{L^{\infty}\left(L^{1}(\Omega)\right)} \leq C \epsilon^{1 / 5},
$$


where $\Omega$ is a bounded domain. Also using this approach, a posteriori error estimates for the equation

$$
u_{t}+\nabla \cdot(\mathbf{v} f(u))-\Delta \varphi(u)=\lambda u
$$

have been obtained (Ohlberger 2001) and adaptivity strategies devised and numerically tested.

So far, there are no a priori error estimates for numerical schemes for degenerate parabolic equations. The first convergence result for monotone schemes for strongly degenerate equations in one space dimension was obtained by Evje and Karlsen (2000b); see also Evje and Karlsen (2000a). Another convergence result for finite volume methods in several space dimensions has been obtained by Eymard, Gallouët, Herbin and Michel (2002b).

\subsection{Hamilton-Jacobi equations}

The theory of entropy solutions of scalar hyperbolic conservation laws runs parallel, in many instances, to the theory of viscosity solutions of HamiltonJacobi equations. For example, the counterpart of the paper by Kružkov (1970) about the entropy solution could be considered to be the papers of Crandall and Lions (1983) and Crandall, Evans and Lions (1984) on the characterization of the viscosity solution.

The error estimate obtained by Kuznetsov (1976) between the entropy solution and the approximation given by a monotone scheme, namely

$$
\left\|u-u_{h}\right\|_{L^{\infty}\left(L^{1}\right)} \leq C\left|u_{0}\right|_{T V} \sqrt{T \Delta x}
$$

corresponds to the error estimate obtained in Crandall and Lions (1984) between the viscosity solution and the approximation given by a monotone scheme, namely,

$$
\left\|u-u_{h}\right\|_{L^{\infty}\left(L^{\infty}\right)} \leq C\left|u_{0}\right|_{W^{1, \infty}} \sqrt{T \Delta x} .
$$

The continuous dependence results on the entropy solution of

$$
u_{t}+\nabla \cdot \mathbf{f}(u)-\Delta \varphi(u)=0
$$

on the nonlinearities in Cockburn and Gripengerg (1999) has as counterpart the continuous dependence results on the viscosity solution of

$$
u_{t}+F\left(u, D_{x} u, D_{x}^{2} u\right)=0,
$$

with respect to the nonlinearity $F$ in Cockburn, Gripengerg and Londen (2001) and Gripenberg (2002); see also Jakobsen and Karlsen (2002).

The counterpart of the a posteriori error estimate in Theorem 6.1 is contained in Albert, Cockburn, French and Peterson (2002a) for the steady state Hamilton-Jacobi equation

$$
u+H(\nabla u)=f
$$


in a periodic setting where a careful numerical study of the effectivity index is carried out. In particular, for monotone schemes and strictly convex Hamiltonians, it is shown that the effectivity index increases like $|\ln \Delta x|$, and not like $(\Delta x)^{-} 1 / 2$ as would be expected if the general theory of monotone schemes is used.

For the transient case, see Albert, Cockburn, French and Peterson (2002b).

The counterpart of the material presented in this paper for entropy solutions is contained in the paper by Cockburn and Qian (2002), where the steady state case was considered.

Finally, the theory of continuous dependence in negative-order norms for the physically relevant solutions of the conservation law

$$
u_{t}+(f(u))_{x}=0
$$

when $f$ is strictly convex, reviewed in Tadmor (1998) has its counterpart in the theory of continuous dependence in $L^{1}$ of viscosity solutions for convex Hamiltonians introduced in Lin and Tadmor (2001).

A result for the Bellman's equation

$$
F\left(D_{t} u, D_{x}^{2} u, D_{x} u, u, x, t\right)=0
$$

that does not have any counterpart in the theory of strongly degenerate convection-diffusion equations is the estimate of the rate of convergence of monotone schemes obtained in Krylov (2000) for fairly general nonlinearities $F$. It reads

$$
-C_{\star}(\Delta x)^{\delta_{\star}} \leq u-u_{h} \leq C^{\star}(\Delta x)^{\delta^{\star}},
$$

where $C_{\star}$ and $C^{\star}$ and positive constants and $\delta_{\star}$ and $\delta_{\star}$ only depend on the smoothness of the coefficients and the function $F$. When $F$ is Lipschitz, we obtain the best estimate, namely

$$
\delta_{\star}=\frac{1}{3} \quad \text { and } \quad \delta^{\star}=\frac{1}{21} .
$$

\section{Concluding remarks and open problems}

In this paper, we have given an overview of a theory of continuous dependence and error estimation for the entropy solution of scalar hyperbolic conservation laws. We have stressed the idea that it is essential to obtain continuous dependence results for the original well-posed problem which do not break down when the viscosity coefficient tends to zero. The existence and uniqueness of the entropy solution are direct consequences of these results, as is the fact that the entropy solution inherits the continuous dependence results valid for the parabolic solutions. This procedure is to be contrasted with the traditional approach of using compactness arguments to obtain the existence of an entropy solution, obtain the so-called entropy 
inequality and only then obtain continuous dependence results. How to extend this approach to more complicated scalar hyperbolic conservation laws, to the corresponding initial boundary value problem, and then to hyperbolic systems remains a challenging open problem.

The other very important open problem is that of studying the minimization of the nonlinear, nonsmooth functional

$$
\Phi(-R(v)),
$$

where $v$ is an approximation of the entropy solution $u$. The devising of iterative adaptive algorithms that guarantee a decrease of the error by a given factor per iteration is certainly the main problem to solve. Strongly

related to this issue is the study of the relation between the upper bound of the error and the error itself,

$$
\frac{\Phi(-R(v))}{\|u-v\|_{L^{\infty}\left(L^{1}\right)}} .
$$

To give an idea of the difficulty of this task, let us recall that this problem has only been recently been solved for finite element approximations of linear strongly elliptic problems in Morin, Nochetto and Siebert (2000). Moreover, in such a case, the functional to minimize was quadratic and smooth!

\section{Acknowledgements}

The author would like to thank Wolfgang Dahmen, Markus Keel, Fernando Reitich and Eitan Tadmor for feedback on the material presented here. The author would also like to thank Kenneth Karlsen for valuable information about degenerate parabolic equations. Finally, the author would like to thank Michael Breuss for a careful reading of the manuscript.

\section{REFERENCES}

R. Abeyaratne and J. Knowles (1991a), 'Implications of viscosity and strain gradient effects for the kinetics of propagating phase boundaries in solids', SIAM J. Appl. Math. 51, 1205-1221.

R. Abeyaratne and J. Knowles (1991b), 'Kinetic relations and the propagation of phase boundaries in solids', Arch. Rational Mech. Anal. 114, 119-154.

S. Albert, B. Cockburn, D. French and T. Peterson (2002a), 'A posteriori error estimates for general numerical methods for Hamilton-Jacobi equations, Part I: The steady state case', Math. Comp. 71, 49-76.

S. Albert, B. Cockburn, D. French and T. Peterson (2002b), A posteriori error estimates for general numerical methods for Hamilton-Jacobi equations, Part II: The time-dependent case, Vol. III of Finite Volumes for Complex Applications, Hermes Penton Science, pp. 17-24.

T. Barth and H. Deconink, eds (1999), High-Order Methods for Computational Physics, Vol. 9 of Lecture Notes in Computational Science and Engineering, Springer. 
P. Benilan and M. Crandall (1981), 'The continuous dependence on $\varphi$ of solutions of $u_{t}-\Delta \varphi(u)=0$ ', Indiana Univ. Math. J. 30, 161-177.

S. Bianchini and A. Bressan (2001), 'Vanishing viscosity solutions of nonlinear hyperbolic systems', Preprint SISSA ref. 86/2001/M November.

F. Bouchut and B. Perthame (1998), 'Kružkov's estimates for scalar conservation laws revisited', Trans. Amer. Math. Soc. 350, 2847-2870.

A. Bressan (2000), Hyperbolic Systems of Conservation Laws: The OneDimensional Cauchy Problem, Vol. 20 of Oxford Lecture Series in Mathematics and its Applications, Oxford University Press, Oxford.

J. Carrillo (1999), 'Entropy solutions for nonlinear degenerate problems', Arch. Rational Mech. Anal. 147, 269-361.

C. Chainais-Hillairet (1999), 'Finite volume schemes for a nonlinear hyperbolic equation: Convergence towards the entropy solution and error estimate', Modél. Math. Anal. Numér. 33, 129-156.

C. Chainais-Hillairet and S. Champier (2001), 'Finite volume schemes for nonhomogeneous scalar conservation laws: Error estimate', Numer. Math. 88, 607-639.

B. Cockburn (1989), 'The quasi-monotone schemes for scalar conservation laws, I', SIAM J. Numer. Anal. 26, 1325-1341.

B. Cockburn (1990a), 'The quasi-monotone schemes for scalar conservation laws, II', SIAM J. Numer. Anal. 27, 247-258.

B. Cockburn (1990b), 'The quasi-monotone schemes for scalar conservation laws, III', SIAM J. Numer. Anal. 27, 259-276.

B. Cockburn (1991), 'On the continuity in $B V(\Omega)$ of the $L^{2}$-projection into finite element spaces', Math. Comp. 57, 551-561.

B. Cockburn (1999), A simple introduction to error estimation for nonnlinear hyperbolic conservation laws: Some ideas, techniques, and promising results, in Proc. 1998 EPSRC Summer School in Numerical Analysis, SSCM, Vol. 26 of The Graduate Student's Guide to Numerical Analysis, Springer, pp. 1-46.

B. Cockburn and H. Gau (1995), 'A posteriori error estimates for general numerical methods for scalar conservation laws', Mat. Aplic. e Comp. 14, 37-45.

B. Cockburn and H. Gau (1996), 'A model numerical scheme for the propagation of phase transitions in solids', SIAM J. Sci. Comput. 17, 1092-1121.

B. Cockburn and P.-A. Gremaud (1996a), 'Error estimates for finite element methods for nonlinear conservation laws', SIAM J. Numer. Anal. 33, 522-554.

B. Cockburn and P.-A. Gremaud (1996b), 'A priori error estimates for numerical methods for scalar conservation laws, Part I: The general approach', Math. Comp. 65, 533-573.

B. Cockburn and P.-A. Gremaud (1997), 'A priori error estimates for numerical methods for scalar conservation laws, Part II: Flux-splitting monotone schemes on irregular Cartesian grids', Math. Comp. 66, 547-572.

B. Cockburn and G. Gripengerg (1999), 'Continuous dependence on the nonlinearities of solutions of degenerate parabolic equations', J. Diff. Eqns 151, $231-251$.

B. Cockburn and J. Qian (2002), Continuous dependence results for HamiltonJacobi equations, in Collected Lectures on the Preservation of Stability under Discretization (D. Estep and S. Tavener, eds), SIAM, pp. 67-90. 
B. Cockburn and C.-W. Shu (2001), 'Runge-Kutta discontinuous Galerkin methods for convection-dominated problems', J. Sci. Comput. 16, 173-261.

B. Cockburn, F. Coquel and P. LeFloch (1994), 'An error estimate for finite volume methods for multidimensional conservation laws', Math. Comp. 63, 77-103.

B. Cockburn, F. Coquel and P. LeFloch (1995), 'Convergence of the finite volume method for multidimensional conservation laws', SIAM J. Numer. Anal. 32, 687-705.

B. Cockburn, P.-A. Gremaud and J. Yang (1998), 'A priori error estimates for hyperbolic conservation laws, Part III: Multidimensional flux-splitting monotone schemes in non-Cartesian grids', SIAM J. Numer. Anal. 35, 1775-1803.

B. Cockburn, G. Karniadakis and C.-W. Shu, eds (2000), Discontinuous Galerkin Methods: Theory, Computation and Applications, Vol. 11 of Lecture Notes in Computational Science and Engineering, Springer.

B. Cockburn, G. Gripengerg and S.-O. Londen (2001), 'Continuous dependence on the nonlinearity of viscosity solutions of parabolic equations', J. Diff. Eqns 170, 180-187.

F. Coquel and P. LeFloch (1991), 'Convergence of finite difference schemes for conservation laws in several space dimensions: The corrected antidiffusive flux approach', Math. Comp. 57, 169-210.

M. Crandall and P. Lions (1983), 'Viscosity solutions of Hamilton-Jacobi equations', Trans. Amer. Math. Soc. 277, 1-42.

M. Crandall and P. Lions (1984), 'Two approximations of solutions of HamiltonJacobi equations', Math. Comp. 43, 1-19.

M. Crandall and A. Majda (1980), 'Monotone difference approximations for scalar conservation laws', Math. Comp. 34, 1-21.

M. Crandall, L. Evans and P. Lions (1984), 'Some properties of viscosity solutions of Hamilton-Jacobi equations', Trans. Amer. Math. Soc. 282, 478-502.

M. Crandall, H. Ishii and P. Lions (1992), 'User's guide to viscosity solutions of second-order partial differential equations', Bull. Amer. Math. Soc. 27, 1-67.

C. Dafermos (2000), Hyperbolic Conservation Laws in Continuum Physics, Vol. 325 of Grundlehren der Mathematischen Wissenschaften (Fundamental Principles of Mathematical Sciences), Springer, Berlin.

R. DeVore and B. Lucier (1990), 'High order regularity for conservation laws', Indiana Univ. Math. J. 39, 413-430.

R. DeVore and B. Lucier (1996), 'On the size and smoothness of solutions to nonlinear hyperbolic conservation laws', SIAM J. Math. Anal. 27, 684-707.

R. DiPerna (1985), 'Measure-valued solutions to conservation laws', Arch. Rat. Mech. Anal. 88, 223-270.

S. Evje and K. Karlsen $(2000 a)$, 'Discrete approximations of $B V$ solutions to doubly nonlinear degenerate parabolic equations', Numer. Math. 86, 377-417.

S. Evje and K. Karlsen (2000b), 'Monotone difference approximations of BV solutions to degenerate convection-diffusion equations', SIAM J. Numer. Anal. 37, 1838-1860.

S. Evje and K. Karlsen (2002), 'An error estimate for viscous approximate solutions of degenerate parabolic equations', J. Nonlin. Math. Phys. 9, 1-20. 
R. Eymard, T. Gallouët, M. Ghilani and R. Herbin (1998), 'Error estimates for the approximate solutions of a nonlinear hyperbolic equation given by finite volume schemes', IMA J. Numer. Anal. 18, 563-594.

R. Eymard, T. Gallouët and R. Herbin (2000), Finite volume methods, in Handbook of Numerical Analysis, Vol. VII, North-Holland, Amsterdam, pp. 713-1020.

R. Eymard, T. Gallouët and R. Herbin (2002a), 'Error estimate for approximate solutions of a nonlinear convection-diffusion problem', Adv. Diff. Eqns 7, 419-440.

R. Eymard, T. Gallouët, R. Herbin and A. Michel (2002b), 'Convergence of a finite volume scheme for nonlinear degenerate parabolic equations', Numer. Math. 92, 41-82.

A. Friedman (1964), Partial Differential Equations of Parabolic Type, Prentice-Hall, Englewood Cliffs, NJ.

E. Godlewski and P.-A. Raviart (1996), Numerical Approximation of Hyperbolic Systems of Conservation Laws, Vol. 118 of Applied Mathematical Sciences, Springer, Paris.

L. Gosse and C. Makridakis (2000), 'Two a posteriori error estimates for onedimensional scalar conservation laws', SIAM J. Numer. Anal. 38, 964-988.

G. Gripenberg (2002), 'Estimates for viscosity solutions of parabolic equations with Dirichlet boundary conditions', Proc. Amer. Math. Soc. 130, 3651-3660.

A. Harten, J. M. Hyman and P. Lax (1976), 'On finite difference approximations and entropy conditions for shocks', Comm. Pure Appl. Math. 29, 297-322.

H. Holden and N. Risebro (2002), Front Tracking for Hyperbolic Conservation Laws, Vol. 152 of Applied Mathematical Sciences, Springer, New York.

D. Jacobs, B. McKinney and M. Shearer (1995), 'Traveling wave solutions of the modified Korteweg-deVries-Burgers equations', J. Diff. Eqns 116, 448-467.

J. Jaffré, C. Johnson and A. Szepessy (1995), 'Convergence of the discontinuous Galerkin finite element method for hyperbolic conservation laws', Math. Models Methods Appl. Sci. 5, 367-386.

E. Jakobsen and K. Karlsen (2002), 'Continuous dependence estimates for viscosity solutions of fully nonlinear degenerate parabolic equations', J. Diff. Eqns 183, 497-525.

C. Johnson (1998), Adaptive finite element methods for conservation laws, in Advanced Numerical Approximation of Nonlinear Hyperbolic Equations (A. Quarteroni, ed.), Vol. 1697 of Lecture Notes in Mathematics; Subseries Fondazione C.I.M.E., Firenze, Springer, pp. 269-323.

D. Kröner (1997), Numerical Schemes for Conservation Laws, Wiley-Teubner series Advances in Numerical Mathematics, Wiley, Chichester.

D. Kröner and M. Ohlberger (2000), 'A posteriori error estimates for upwind finite volume schemes for nonlinear conservation laws in multidimensions', Math. Comp. 69, 25-39.

D. Kröner and M. Rokyta (1994), 'Convergence of upwind finite volume methods for scalar conservation laws in two dimensions', SIAM J. Numer. Anal. 31, $324-343$.

D. Kröner, S. Noelle and M. Rokyta (1995), 'Convergence of higher order upwind finite volume schemes on unstructured grids for scalar conservation laws in several space dimensions', Numer. Math. 71, 527-560. 
D. Kröner, M. Ohlberger and C. Rohde, eds (1999), An Introduction to Recent Developments in Theory and Numerics for Conservation Laws, Vol. 5 of Lecture Notes in Computational Science and Engineering, Springer, Berlin. (Papers from the International School on Theory and Numerics for Conservation Laws held in Freiburg/Littenweiler, October 20-24, 1997.)

S. N. Kružkov (1970), 'First order quasilinear equations in several independent variables', Math. USSR-Sb 10, 217-243.

N. Krylov (2000), 'SPDEs in $L_{Q}\left((0, \tau], L_{P}\right)$ spaces', Electron. J. Probab. 5, \#13.

N. N. Kuznetsov (1976), 'Accuracy of some approximate methods for computing the weak solutions of a first-order quasi-linear equation', USSR Comp. Math. Math. Phys. 16, 105-119.

P. Lax (1972), Hyperbolic Systems of Conservation Laws and the Mathematical Theory of Shock waves, Vol. 11 of CMBS Regional Conference Series in Applied Mathematics, SIAM, Philadephia.

R. J. LeVeque (1990), Numerical Methods for Conservation Laws, Birkhäuser.

C.-T. Lin and E. Tadmor (2001), ' $L^{1}$-stability and error estimates for approximate Hamilton-Jacobi solutions', Numer. Math. 87, 701-735.

T.-P. Liu (1997), Hyperbolic and Viscous Systems of Conservation Laws, Vol. 18 of CMBS Regional Conference Series in Applied Mathematics, SIAM, Philadephia.

B. J. Lucier (1985a), 'Error bounds for the methods of Glimm, Godunov and LeVeque', SIAM J. Numer. Anal. 22, 1074-1081.

B. J. Lucier (1985b), 'A stable adaptive scheme for hyperbolic conservation laws', SIAM J. Numer. Anal. 22, 180-203.

B. J. Lucier (1986), 'A moving mesh numerical method for hyperbolic conservation laws', Math. Comp. 46, 59-69.

B. J. Lucier (1988), 'Regularity through approximation for scalar conservation laws', SIAM J. Numer. Anal. 19, 763-773.

B. J. Lucier (200x), Numerical Analysis of Conservation Laws. In preparation.

B. J. Lucier and R. Overbeek (1987), 'A parallel adaptive numerical scheme for hyperbolic systems of conservation laws', SIAM J. Sci. Statist. Comput. 8, S203-S219.

J. Málek, J. Nečas, M. Rokyta and M. Ružička (1996), Weak and Measure-Valued Solutions to Evolutionary PDEs, Vol. 13 of Applied Mathematics and Mathematical Computation, Chapman \& Hall, London.

C. Mascia, A. Porretta and A. Terracina (2002), 'Non-homogeneous Dirichlet problems for degenerate parabolic-hyperbolic equations', Arch. Rational Mech. Anal. 163, 87-124.

P. Morin, R. Nochetto and K. Siebert (2000), 'Data oscillation and convergence of adaptive FEM', SIAM J. Numer. Anal. 38, 466-488.

S. Nöelle (1996), 'A note on entropy inequalities and error estimates for higherorder accurate finite volume schemes on irregular families of grids', Math. Comp. 65, 1155-1163.

S. Nöelle (1995), 'Convergence of higher order finite volume schemes on irregular grids', Adv. Comput. Math. 3, 197-218. 
M. Ohlberger (2001), 'A posteriori error estimates for vertex centered finite volume approximations of convection-diffusion-reaction equations', Modél. Math. Anal. Numér. 35, 355-387.

J. Rauch (1986), 'BV estimates fail for most quasilinear hyperbolic systems in dimensions greater than one', Commun. Math. Phys. 106, 481-484.

F. Şabac (1997), 'The optimal convergence rate of monotone finite difference methods for hyperbolic conservation laws', SIAM J. Numer. Anal. 34, 2306-2318.

R. Sanders (1983), 'On convergence of monotone finite difference schemes with variable spacing differencing', Math. Comp. 40, 91-106.

D. Serre (1999), Systems of Conservation Laws, 1: Hyperbolicity, Entropies, Shock Waves, Cambridge University Press, Cambridge. Translated from the 1996 French original by I. N. Sneddon.

D. Serre (2000), Systems of Conservation Laws, 2: Geometric Structures, Oscillations, and Initial-Boundary Value Problems, Cambridge University Press, Cambridge. Translated from the 1996 French original by I. N. Sneddon.

C.-W. Shu (1992), 'A numerical method for systems of conservation laws of mixed type admitting hyperbolic flux splitting', J. Comput. Phys. 100, 424-429.

C.-W. Shu (1998), Essentially non-oscillatory and weighted essentially nonoscillatory schemes for hyperbolic conservation laws, in Advanced Numerical Approximation of Nonlinear Hyperbolic Equations (A. Quarteroni, ed.), Vol. 1697 of Lecture Notes in Mathematics: Subseries Fondazione C.I.M.E., Firenze, Springer, pp. 325-432.

M. Slemrod (1984), 'Dynamic phase transitions in a van der Waals fluid', J. Diff. Eqns 52, 1-23.

M. Slemrod and J. Flaherty (1986), Numerical integration of a Riemman problem for a van der Waals fluid, in Phase Transformations (E. Aifantis and J. Gittus, eds), Elsevier Applied Science, pp. 203-212.

A. Szepessy (1989), 'Convergence of a shock-capturing streamline-diffusion finite element method for scalar conservation laws in two space dimensions', Math. Comp. 53, 527-545.

A. Szepessy (1991), 'Convergence of a streamline-diffusion finite element method for a conservation law with boundary conditions', RAIRO Modél. Math. Anal. Numér. 25, 749-783.

E. Tadmor (1998), Approximate solutions of nonlinear conservation laws, in Advanced Numerical Approximation of Nonlinear Hyperbolic Equations (A. Quarteroni, ed.), Vol. 1697 of Lecture Notes in Mathematics: Subseries Fondazione C.I.M.E., Firenze, Springer, pp. 1-149.

E. Toro (1997), Riemann Solvers and Numerical Methods for Fluid Dynamics, Springer.

L. Truskinovsky (1982), 'Equilibrium phase interfaces', Dokl. Akad. Nauk. SSSR 256, 306-310.

J.-P. Vila (1994), 'Convergence and error estimates for finite volume schemes for general multidimensional scalar conservation laws', Model. Math. Anal. Numér. 28, 267-295.

G. B. Witham (1974), Linear and Nonlinear Waves, Wiley, New York.

X. Zhong, T. Hou and P. LeFloch (1996), 'Computational methods for propagating phase boundaries', J. Comput. Phys. 124, 192-216. 


\section{Appendix: Proofs of some results}

In this appendix, we sketch the proofs of some of the results in this paper.

Proof of Proposition 4.3 (a priori error estimates for parabolic solutions). Property (i) follows from Theorem 4.2 by first taking $v(x, t)=a(t), s(t)=$ $-\sup _{x \in \mathbb{R}^{d}} \max \{-r(x, t), 0\}$ and $U(e)=\max \{-e, 0\}$, and then $v(x, t)=b(t)$, $s(t)=\sup _{x \in \mathbb{R}^{d}} \max \{r(x, t), 0\}$ and $U(e)=\max \{e, 0\}$.

Property (ii) is obtained by proving that, for each $i=1, \ldots, d$, we have

$$
\left\|\partial_{x_{i}} u(T)\right\|_{L^{1}} \leq\left\|\partial_{x_{i}} u_{0}\right\|_{L^{1}}+\left\|\partial_{x_{i}} r\right\|_{L^{1}\left(L^{1}\right)},
$$

and then summing over $i$. To prove the above inequality, we take, in Theorem 4.2, $v(x, t)=u\left(x+h e_{i}, t\right), s(x, t)=r\left(x+h e_{i}, t\right)$, and $U(e)=|e|$, where $e_{i}$ is the $i$ th canonical vector in $\mathbb{R}^{d}$. The result follows after dividing by $|h|$ and letting $h$ tend to zero.

This completes the proof.

Proof of Proposition 4.4 (continuous dependence results for parabolic solutions). Property (i) follows from Theorem 4.2 by taking $s=r$ and $U(e)=$ $\max \{-e, 0\}$, and property (ii) by taking $s=r$ and $U(e)=|e|$. To obtain property (iii), we begin by taking $v(t)=u(t+\delta t$ ) in (ii), dividing by $\delta t>0$ and letting it tend to zero, obtaining

$$
\left\|u_{t}(T)\right\|_{L^{1}} \leq\left\|u_{t}(0)\right\|_{L^{1}}+\left\|r_{t}\right\|_{L^{1}\left(L^{1}\right)} .
$$

Then we use the estimate

$$
\left\|u_{t}(0)\right\|_{L^{1}} \leq\|\mathbf{f}\|_{L^{\infty}(I)}\left|u_{0}\right|_{T V}+\nu\left\|\Delta u_{0}\right\|_{L^{1}} .
$$

Finally, property (iv) follows by taking $u=u_{1}, v=u_{2}$ and $s=r+\mathbf{f}_{1}\left(u_{2}\right)-$ $\mathbf{f}_{2}\left(u_{2}\right)$.

Proof of Theorem 5.1 (continuous dependence for parabolic equations with the doubling of the space variable technique). One way to introduce the technique of doubling the space variable is to consider working with the convolution $\varphi_{\epsilon_{x}} * u$, because this regularization inherits the smoothness of the convolution kernel $\varphi_{\epsilon_{x}}$; we would then avoid the problems associated with the appearance of strong gradients as the viscosity coefficient becomes smaller and smaller.

Our objective would now be to estimate

$$
\left|\varphi_{\epsilon_{x}} * u-v\right|_{U}
$$

but it is preferable to symmetrize the role of $u$ and $v$ and work instead with the functional

$$
\langle u, v\rangle_{U}^{\epsilon_{x}}:=\int_{\mathbb{R}^{d} \times \mathbb{R}^{d}} \varphi_{\epsilon_{x}}(x-y) U(u(x)-v(y)) \mathrm{d} y \mathrm{~d} x .
$$


Indeed, not only do we have that

$$
\max \left\{\left|\varphi_{\epsilon_{x}} * u-v\right|_{U},\left|u-\varphi_{\epsilon_{x}} * v\right|_{U}\right\} \leq\langle u, v\rangle_{U}^{\epsilon_{x}}
$$

for all convex, nonnegative functions $U$, but also

$$
|| u-\left.v\right|_{U}-\langle u, v\rangle_{U}^{\epsilon_{x}} \mid \leq\langle u, u\rangle_{U}^{\epsilon_{x}} \leq \mathrm{c}_{1} \epsilon_{x}\|\nabla u\|_{L^{1}},
$$

for $U$, as in (4.5).

We thus see that an estimate on $\langle u, v\rangle_{U}^{\epsilon_{x}}$ immediately implies an estimate on $|u-v|_{U}$. We also see that the introduction of the convolution naturally leads to the so-called doubling of the variables technique, as now $u=u(x, t)$ and $v=v(y, t)$ depend on different space variables.

To prove Theorem 5.1, we begin by obtaining the following extension of Lemma 4.1.

Lemma A1. Let $u$ be the solution of (4.1) and (4.2) and let $v$ be the solution of (5.3) and (5.4). Let $U$ be any smooth function such that $U(0)=0$. Then,

$$
\langle u(T), v(T)\rangle_{U}^{\epsilon_{x}}+\Theta_{U}^{\epsilon_{x}}=\left\langle u_{0}, v_{0}\right\rangle_{U}^{\epsilon_{x}}+V_{U}^{\epsilon_{x}}+R_{U}^{\epsilon_{x}}
$$

where

$$
\begin{aligned}
& \Theta_{U}^{\epsilon_{x}}=\int_{0}^{T} \int_{\mathbb{R}^{d} \times \mathbb{R}^{d}} \varphi_{\epsilon_{x}}(x-y)\left(Y_{\mathbf{f}, U, \epsilon_{x}}+Z_{\nu, U, \epsilon_{x}}\right) \mathrm{d} x \mathrm{~d} y \mathrm{~d} t \\
& V_{U}^{\epsilon_{x}}=\left(\sqrt{\nu_{1}}-\sqrt{\nu_{2}}\right)^{2} \int_{0}^{T} \int_{\mathbb{R}^{d} \times \mathbb{R}^{d}} U(u-v) \Delta_{y} \varphi_{\epsilon_{x}}(x-y) \mathrm{d} x \mathrm{~d} y \mathrm{~d} t \\
& R_{U}^{\epsilon_{x}}=\int_{0}^{T} \int_{\mathbb{R}^{d} \times \mathbb{R}^{d}} \varphi_{\epsilon_{x}}(x-y) U^{\prime}(u-v)(r-s) \mathrm{d} x \mathrm{~d} y \mathrm{~d} t
\end{aligned}
$$

and

$$
\begin{aligned}
Y_{\mathbf{f}, U} & =\nabla_{y} v \cdot \int_{v}^{u} U^{\prime \prime}(w-v)\left(\mathbf{f}^{\prime}(w)-\mathbf{f}^{\prime}(v)\right) \mathrm{d} w, \\
Z_{\nu, U, \epsilon_{x}} & =\left|\sqrt{\nu} \nabla_{x} u-\sqrt{\nu^{\prime}} \nabla_{y} v\right|^{2} U^{\prime \prime}(u-v) .
\end{aligned}
$$

In the above expressions, $u=u(x, t), r=r(x, t), v=v(y, t)$ and $s=s(y, t)$.

Proof. Let us begin by pointing out that, since we have doubled the variables, we indicate that we are differentiating with respect to the variable $x$ by the sub-index ' $x$ ', and with respect to the variable $y$ by the sub-index ' $y$ '. Thus, we rewrite equations (4.1) and (5.3) as follows:

$$
\begin{aligned}
& u_{t}+\nabla_{x} \cdot \mathbf{f}(u)-\nu \Delta_{x} u=r \\
& v_{t}+\nabla_{y} \cdot \mathbf{f}(v)-\nu^{\prime} \Delta_{y} v=s
\end{aligned}
$$

Subtracting the second equation from the first and multiplying by $U^{\prime}(u-v)$, 
we get

$$
(U(u-v))_{t}+C_{\mathbf{f}}+D_{\nu}=U^{\prime}(u-v)(r-s)
$$

where

$$
\begin{aligned}
C_{\mathbf{f}} & =U^{\prime}(u-v)\left(\nabla_{x} \cdot \mathbf{f}(u)-\nabla_{y} \cdot \mathbf{f}(v)\right), \\
D_{\nu} & =U^{\prime}(u-v)\left(\nu \Delta_{x} u-\nu^{\prime} \Delta_{y} v\right) .
\end{aligned}
$$

Next, we rewrite the above expression by using the fact that $u=u(x, t)$ does not depend on $y$ and that $v=v(y, t)$ does not depend on $x$.

Let us begin with the term associated with convection, $C_{\mathbf{f}}$. Since

$$
\begin{aligned}
U^{\prime}(u-v) \nabla_{x} \cdot \mathbf{f}(u) & =\nabla_{x} \cdot \mathbf{F}(u, v), \\
-U^{\prime}(u-v) \nabla_{y} \cdot \mathbf{f}(v) & =\nabla_{y} \cdot \mathbf{G}(u, v),
\end{aligned}
$$

where

$$
\begin{aligned}
\mathbf{F}(u, v) & =\int_{v}^{u} U^{\prime}(w-v) \mathbf{f}^{\prime}(w) \mathrm{d} w \\
\mathbf{G}(u, v) & =\int_{v}^{u} U^{\prime}(u-w) \mathbf{f}^{\prime}(w) \mathrm{d} w
\end{aligned}
$$

we obtain that

$$
\begin{aligned}
C_{\mathbf{f}} & =\nabla_{x} \cdot \mathbf{F}(u, v)+\nabla_{y} \cdot \mathbf{G}(u, v) \\
& =\left(\nabla_{x}+\nabla_{y}\right) \cdot \mathbf{F}(u, v)+\nabla_{y} \cdot(\mathbf{G}(u, v)-\mathbf{F}(u, v)) \\
& =\left(\nabla_{x}+\nabla_{y}\right) \cdot \mathbf{F}(u, v)+Y_{\mathbf{f}, U} .
\end{aligned}
$$

Now let us work on the term associated to diffusion, $D_{\nu}$. Since

$$
\begin{aligned}
U^{\prime}(u-v) \Delta_{x} u & =\Delta_{x} U(u-v)-U^{\prime \prime}(u-v)\left|\nabla_{x} u\right|^{2}, \\
-U^{\prime}(u-v) \Delta_{y} v & =\Delta_{y} U(u-v)-U^{\prime \prime}(u-v)\left|\nabla_{y} v\right|^{2},
\end{aligned}
$$

we get that

$$
D_{\nu}=\left(\nu \Delta_{x}+\nu^{\prime} \Delta_{y}\right) U(u-v)+U^{\prime \prime}(u-v)\left(\nu\left|\nabla_{x} u\right|^{2}+\nu^{\prime}\left|\nabla_{y} v\right|^{2}\right) .
$$

Completing squares in the last term of the right-hand side by adding and subtracting the expression

$$
2 \sqrt{\nu \nu^{\prime}} U^{\prime \prime}(u-v) \nabla_{x} u \cdot \nabla_{y} v=-2 \sqrt{\nu \nu^{\prime}} \nabla_{x} \cdot \nabla_{y} U(u-v)
$$

we obtain

$$
D_{\nu}=\mathbb{L}_{\nu} U(u-v)+Z_{\nu, U},
$$

where

$$
\mathbb{L}_{\nu}=\nu \Delta_{x}+2 \sqrt{\nu \nu^{\prime}} \nabla_{x} \cdot \nabla_{y}+\nu^{\prime} \Delta_{y} .
$$

As a consequence, we get

$$
\begin{aligned}
(U(u-v))_{t}+ & \left(\nabla_{x}+\nabla_{y}\right) \cdot \mathbf{F}(u, v)+Y_{\mathbf{f}, U} \\
& -\mathbb{L}_{\nu} U(u-v)+Z_{\nu, U}=U^{\prime}(u-v)(r-s) .
\end{aligned}
$$


Now we multiply the above equation by the convolution kernel $\varphi_{\epsilon_{x}}(x-y)$ and integrate over $\mathbb{R}^{d} \times \mathbb{R}^{d}$ the on $(0, T)$. We get

$$
\langle u(T), v(T)\rangle_{U}^{\epsilon_{x}}+\Theta_{U}^{\epsilon_{x}}+\widehat{C}_{\mathbf{f}}=\left\langle u_{0}, v_{0}\right\rangle_{U}^{\epsilon_{x}}+\widehat{D}_{\nu}+R_{U}^{\epsilon_{x}}
$$

where

$$
\begin{aligned}
& \widehat{C}_{\mathbf{f}}=\int_{0}^{T} \int_{\mathbb{R}^{d} \times \mathbb{R}^{d}} \varphi_{\epsilon_{x}}(x-y)\left(\nabla_{x}+\nabla_{y}\right) \cdot \mathbf{F}(u, v) \mathrm{d} x \mathrm{~d} y \mathrm{~d} t \\
& \widehat{D}_{\nu}=\int_{0}^{T} \int_{\mathbb{R}^{d} \times \mathbb{R}^{d}} \varphi_{\epsilon_{x}}(x-y) \mathbb{L}_{\nu} U(u-v) \mathrm{d} x \mathrm{~d} y \mathrm{~d} t .
\end{aligned}
$$

After a simple integration by parts, we obtain that $\widehat{C}_{\mathbf{f}}=0$ and $\widehat{D}_{\nu}=V_{U}^{\epsilon_{x}}$ since

$$
\left(\nabla_{x}+\nabla_{y}\right) \varphi_{\epsilon_{x}}(x-y)=0, \quad \mathbb{L}_{\nu} \varphi_{\epsilon_{x}}(x-y)=\left(\sqrt{\nu}-\sqrt{\nu^{\prime}}\right)^{2} \Delta_{y} \varphi_{\epsilon_{x}}(x-y) .
$$

This completes the proof.

We can now prove Theorem 5.1. We have, by (A.2),

$$
\begin{aligned}
|u(T)-v(T)|_{U} & \leq\langle u(T), v(T)\rangle_{U}^{\epsilon_{x}}+c_{1} \epsilon_{x} \min \left\{\|u(T)\|_{T V},\|v(T)\|_{T V}\right\}, \\
\left\langle u_{0}, v_{0}\right\rangle_{U}^{\epsilon_{x}} & \leq\left|u_{0}-v_{0}\right|_{U}+c_{1} \epsilon_{x} \min \left\{\left\|u_{0}\right\|_{T V},\left\|v_{0}\right\|_{T V}\right\} .
\end{aligned}
$$

Hence

$$
|u(T)-v(T)|_{U} \leq\left|u_{0}-v_{0}\right|_{U}+c_{1} \epsilon_{x} \mathrm{~A}+\langle u(T), v(T)\rangle_{U}^{\epsilon_{x}}-\left\langle u_{0}, v_{0}\right\rangle_{U}^{\epsilon_{x}} .
$$

Then, by Lemma A1, with $U$ given by (4.5),

$$
|u(T)-v(T)|_{U} \leq\left|u_{0}-v_{0}\right|_{U}+c_{1} \epsilon_{x} \mathrm{~A}+V_{U}^{\epsilon_{x}}+R_{U}^{\epsilon_{x}} .
$$

Next, let us estimate $V_{U}^{\epsilon_{x}}$. Integrating by parts, we get

$$
V_{U}^{\epsilon_{x}}=\left(\sqrt{\nu}-\sqrt{\nu^{\prime}}\right)^{2} \int_{0}^{T} \int_{\mathbb{R}^{d} \times \mathbb{R}^{d}} U^{\prime}(u-v) \nabla_{y} v \cdot \nabla_{y} \varphi_{\epsilon_{x}}(x-y) \mathrm{d} x \mathrm{~d} y \mathrm{~d} t
$$

where $u=u(x, t)$ and $v=v(y, t)$, and since $\left|U^{\prime}\right| \leq 1$,

$$
V_{U}^{\epsilon_{x}} \leq\left(\sqrt{\nu}-\sqrt{\nu^{\prime}}\right)^{2} \frac{\mathrm{c}_{2}}{\epsilon_{x}}\|v\|_{L^{1}(T V)} .
$$

Similarly, since $\Delta_{y} \varphi_{\epsilon_{x}}(x-y)=\Delta_{x} \varphi_{\epsilon_{x}}(x-y)$, we obtain

$$
V_{U}^{\epsilon_{x}} \leq\left(\sqrt{\nu}-\sqrt{\nu^{\prime}}\right)^{2} \frac{c_{2}}{\epsilon_{x}}\|u\|_{L^{1}(T V)} .
$$

Finally, we have, for $U$ given by (4.5),

$$
R_{U}^{\epsilon_{x}}=\int_{0}^{T} \int_{\mathbb{R}^{d} \times \mathbb{R}^{d}} \varphi_{\epsilon_{x}}(x-y) U^{\prime}(u-v)(r(x, t)-s(y, t)) \mathrm{d} y \mathrm{~d} x \mathrm{~d} t \leq \Lambda_{U}^{\epsilon_{x}}(v) .
$$

This completes the proof. 
Proof of Theorem 6.1 (continuous dependence with the doubling of the space and time variables). The only difference between the technique of doubling the space variable and doubling the time variable is associated with the treatment of the boundaries of the time domain, $(0, T)$. We display this difference on the model equations

$$
\begin{array}{lll}
u_{t}=F(t) & \forall t \in(0, T), & u(0)=u_{0}, \\
v_{\tau}=G(\tau) & \forall \tau \in(0, T), & v(0)=v_{0} .
\end{array}
$$

Lemma A2. For $U$ given by (4.5), we have

$$
|u(T)-v(T)|_{U} \leq\left|u_{0}-v_{0}\right|_{U}+\mathrm{D} \epsilon_{t}+\Theta_{U}^{\epsilon_{t}},
$$

where

$$
\begin{aligned}
\mathrm{D} & =\left|u_{t}\right|_{L^{\infty}(0, T)}+\left|v_{t}\right|_{L^{\infty}(0, T)}, \\
\Theta_{U}^{\epsilon_{t}} & =\frac{1}{\Phi_{\epsilon_{t}}(T)} \int_{0}^{T} \int_{0}^{T} \varphi_{\epsilon_{t}}(t-\tau) U^{\prime}(u(t)-v(\tau))(F(t)-G(\tau)) \mathrm{d} \tau \mathrm{d} t .
\end{aligned}
$$

Proof. Let $U$ be any smooth function. Then

$$
\left(\partial_{t}+\partial_{\tau}\right) U(u(t)-v(\tau))=U^{\prime}(u(t)-v(\tau))(F(t)-G(\tau)) .
$$

Multiplying by $\varphi_{\epsilon_{t}}(t-\tau)$ and using the fact that $\left(\partial_{t}+\partial_{\tau}\right) \varphi_{\epsilon_{t}}(t-\tau)=0$, we get

$$
D_{u}+D_{v}=\Phi_{\epsilon_{t}}(T) \Theta_{U}^{\epsilon_{t}}
$$

where

$$
\begin{aligned}
& D_{u}=\left.\int_{0}^{T} \varphi_{\epsilon_{t}}(t-\tau) U(u(t)-v(\tau)) \mathrm{d} \tau\right|_{t=0} ^{t=T}, \\
& D_{v}=\left.\int_{0}^{T} \varphi_{\epsilon_{t}}(t-\tau) U(u(t)-v(\tau)) \mathrm{d} t\right|_{\tau=0} ^{\tau=T} .
\end{aligned}
$$

Now, note that, for $U$ given by (4.5), the triangle inequality gives

$$
\begin{array}{r}
\int_{0}^{T} \varphi_{\epsilon_{t}}(T-\tau) U(u(T)-v(\tau)) \mathrm{d} \tau \geq \frac{1}{2} \Phi_{\epsilon_{t}}(T) U(u(T)-v(T)) \\
-\frac{\epsilon_{t}}{2} \Phi_{\epsilon_{t}}(T)\left|v_{t}\right|_{L^{\infty}(0, T)} .
\end{array}
$$

In a similar manner, the following inequalities are obtained:

$$
\begin{aligned}
D_{u} & \geq \frac{1}{2} \Phi_{\epsilon_{t}}(T)\left(U(u(T)-v(T))-U\left(u_{0}-v_{0}\right)\right)-\Phi_{\epsilon_{t}}(T)\left|v_{t}\right|_{L^{\infty}(0, T)}, \\
D_{v} & \geq \frac{1}{2} \Phi_{\epsilon_{t}}(T)\left(U(u(T)-v(T))-U\left(u_{0}-v_{0}\right)\right)-\Phi_{\epsilon_{t}}(T)\left|u_{t}\right|_{L^{\infty}(0, T)} .
\end{aligned}
$$

This completes the proof. 
Using the above lemma, we prove Theorem 6.1. If $v$ is the solution of (5.3) and (5.4), then it is not difficult to combine Lemma A2 with the proof of Theorem 5.1, to obtain

$$
\begin{aligned}
\mid u(T) & -\left.v(T)\right|_{U} \\
& \leq\left|u_{0}-v_{0}\right|_{U}+\inf _{\epsilon_{x}, \epsilon_{t}>0}\left(\mathrm{~A} \epsilon_{x}+\frac{\left(\sqrt{\nu}-\sqrt{\nu^{\prime}}\right)^{2}}{\epsilon_{x}} \mathrm{~B}+\mathrm{C} \epsilon_{t}+\Lambda_{U}^{\epsilon_{x}, \epsilon_{t}}(v)\right),
\end{aligned}
$$

where

$$
\Lambda_{U}^{\epsilon_{x}, \epsilon_{t}}(v)=\frac{1}{\Phi_{\epsilon_{t}}(T)} \int_{0}^{T} \int_{\mathbb{R}^{d}} \mathcal{E}_{U}^{\epsilon_{x}, \epsilon_{t}}(v, u ; x, t) \mathrm{d} x \mathrm{~d} t
$$

and

$$
\mathcal{E}_{U}^{\epsilon_{x}, \epsilon_{t}}(v, u ; x, t)=\int_{0}^{T} \int_{\mathbb{R}^{d}} \varphi_{\epsilon_{t}, \epsilon_{x}} U^{\prime}(u-v)(r-s) \mathrm{d} y \mathrm{~d} \tau .
$$

Now, note that we can write

$U^{\prime}(u-v)(r-s)=U^{\prime}(u-v) r+(U(u-v))_{\tau}+\nabla_{y} \cdot \mathbf{G}(u, v)+\nu^{\prime} U^{\prime}(u-v) \Delta_{y} v$, and so

$$
\begin{aligned}
\mathcal{E}_{U}^{\epsilon_{x}, \epsilon_{t}}(v, u ; x, t)= & \left.\int_{\mathbb{R}^{d}} \varphi_{\epsilon_{t}, \epsilon_{x}} U(u-v) \mathrm{d} y\right|_{\tau=0} ^{\tau=T}-\int_{0}^{T} \int_{\mathbb{R}^{d}} U(u-v)\left(\varphi_{\epsilon_{t}, \epsilon_{x}}\right)_{t} \mathrm{~d} y \mathrm{~d} \tau \\
& -\int_{0}^{T} \int_{\mathbb{R}^{d}}\left(\mathbf{G}(u, v) \cdot \nabla_{y} \varphi_{\epsilon_{t}, \epsilon_{x}}-U^{\prime}(u-v) r \varphi_{\epsilon_{t}, \epsilon_{x}}\right) \mathrm{d} y \mathrm{~d} \tau \\
& +\nu^{\prime} \int_{0}^{T} \int_{\mathbb{R}^{d}} U^{\prime}(u-v) \Delta_{y} v \varphi_{\epsilon_{t}, \epsilon_{x}} \mathrm{~d} y \mathrm{~d} \tau \\
\leq & \sup _{c \in \mathbb{R}} E_{U}^{\epsilon_{x}, \epsilon_{t}}(v, c ; x, t)+\nu^{\prime} \int_{0}^{T} \int_{\mathbb{R}^{d}} U^{\prime}(u-v) \Delta_{y} v \varphi_{\epsilon_{t}, \epsilon_{x}} \mathrm{~d} y \mathrm{~d} \tau .
\end{aligned}
$$

If we let $\nu^{\prime}$ and then $\nu$ tend to zero, we obtain the result for smooth $v$. Now the result follows from a classical density argument. This completes the proof.

Proof of Theorem 6.2 (continuous dependence with a variation of the doubling of the time-variable technique).

Lemma A3. For $U$ given by (4.5), we have

$$
|u(T)-v(T)|_{U} \leq 2\left|u_{0}-v_{0}\right|_{U}+4 \mathrm{D} \epsilon_{t}+4 \sup _{z \in\left[0, \epsilon_{t}\right] \cup\{T\}} \Theta_{U}^{\epsilon_{t}}(z),
$$

where

$$
\begin{aligned}
\mathrm{D} & =2 \min \left\{\left|u_{t}\right|_{L^{\infty}(0, T)},\left|v_{t}\right|_{L^{\infty}(0, T)}\right\} \\
\Theta_{U}^{\epsilon_{t}}(z) & =\frac{1}{\Phi_{\epsilon_{t}}(z)} \int_{0}^{z} \int_{0}^{z} \varphi_{\epsilon_{t}}(t-\tau) U^{\prime}(u(t)-v(\tau))(F(t)-G(\tau)) \mathrm{d} \tau \mathrm{d} t .
\end{aligned}
$$


Proof. We proceed exactly as in the proof of Lemma A2, to get

$$
D_{u}+D_{v}=\Phi_{\epsilon_{t}}(T) \Theta_{U}^{\epsilon_{t}} .
$$

Next, we estimate $D_{u}$ in a different way. Setting $e(t)=U(u(t)-v(t))$, we get

$$
\begin{aligned}
\int_{0}^{T} \varphi_{\epsilon_{t}}(T-\tau) U(u(T)-v(\tau)) \mathrm{d} \tau \geq & \int_{0}^{T} \varphi_{\epsilon_{t}}(T-\tau) e(\tau) \mathrm{d} \tau \\
& -\frac{\epsilon_{t}}{2} \Phi_{\epsilon_{t}}(T)\left|u_{t}\right|_{L^{\infty}(0, T)}, \\
\int_{0}^{T} \varphi_{\epsilon_{t}}(0-\tau) U\left(u_{0}-v(\tau)\right) \mathrm{d} \tau \leq & \int_{0}^{T} \begin{array}{l}
\varphi_{\epsilon_{t}}(\tau) e(\tau) \mathrm{d} \tau \\
+\frac{\epsilon_{t}}{2} \Phi_{\epsilon_{t}}(T)\left|u_{t}\right|_{L^{\infty}(0, T)}
\end{array}
\end{aligned}
$$

As a consequence, we obtain

$$
\begin{aligned}
D_{u} & \geq \int_{0}^{T}\left(\varphi_{\epsilon_{t}}(T-\tau)-\varphi_{\epsilon_{t}}(\tau)\right) e(\tau) \mathrm{d} \tau-\epsilon_{t} \Phi_{\epsilon_{t}}(T)\left|u_{t}\right|_{L^{\infty}(0, T)}, \\
D_{v} & \left.\geq \frac{1}{2} \Phi_{\epsilon_{t}}(T)(e(T))-e(0)\right)-\epsilon_{t} \Phi_{\epsilon_{t}}(T)\left|u_{t}\right|_{L^{\infty}(0, T)} .
\end{aligned}
$$

In a similar way, we get

$$
\begin{aligned}
& \left.D_{u} \geq \frac{1}{2} \Phi_{\epsilon_{t}}(T)(e(T))-e(0)\right)-\epsilon_{t} \Phi_{\epsilon_{t}}(T)\left|v_{t}\right|_{L^{\infty}(0, T)} \\
& D_{v} \geq \int_{0}^{T}\left(\varphi_{\epsilon_{t}}(T-\tau)-\varphi_{\epsilon_{t}}(\tau)\right) e(\tau) \mathrm{d} \tau-\epsilon_{t} \Phi_{\epsilon_{t}}(T)\left|v_{t}\right|_{L^{\infty}(0, T)} .
\end{aligned}
$$

As a consequence,

$$
e(T)+\frac{2}{\Phi_{\epsilon_{t}}(T)} \int_{0}^{T} \varphi_{\epsilon_{t}}(T-\tau) e(\tau) \mathrm{d} \tau \leq \zeta(T)+\frac{2}{\Phi_{\epsilon_{t}}(T)} \int_{0}^{T} \varphi_{\epsilon_{t}}(\tau) e(\tau) \mathrm{d} \tau
$$

where

$$
\zeta(T)=e(0)+2 \epsilon_{t} \mathrm{D}+2 \Theta_{U}^{\epsilon_{t}}(T) .
$$

Thus, if $T \leq \epsilon_{t}, \varphi_{\epsilon_{t}}(T-\tau)=\varphi_{\epsilon_{t}}(\tau)$ for all $\tau \in[0, T]$, and so

$$
e(T) \leq \zeta(T) .
$$

Now, if $T \geq \epsilon_{t}$,

$$
e(T) \leq \zeta(T)+\sup _{\tau \in\left(0, \epsilon_{t}\right)} e(\tau) \leq 2 \sup _{z \in\left[0, \epsilon_{t}\right] \cup\{T\}} \zeta(z),
$$

and the result follows. This completes the proof. 
Proof of Corollary 7.1 (characterization of piecewise smooth entropy solutions). By Theorem 6.1, with $U(e)=|e|$, we have

$$
\|u(T)-v(T)\|_{L^{1}} \leq\left\|u_{0}-v_{0}\right\|_{L^{1}}+\inf _{\epsilon_{x}, \epsilon_{t}>0}\left(\mathrm{~A} \epsilon_{x}+\mathrm{C} \epsilon_{t}+\Lambda_{U}^{\epsilon_{x}, \epsilon_{t}}(v)\right),
$$

where

$$
\Lambda_{U}^{\epsilon_{x}, \epsilon_{t}}(v)=\frac{1}{\Phi_{\epsilon_{t}}(T)} \int_{0}^{T} \int_{\mathbb{R}} \sup _{c \in \mathbb{R}} E_{U}^{\epsilon_{x}, \epsilon_{t}}(v, c ; x, t) \mathrm{d} x \mathrm{~d} t,
$$

and, in our case, $E_{U}^{\epsilon_{x}, \epsilon_{t}}(v, c ; x, t)$ is equal to

$$
\begin{array}{r}
\int_{\Omega^{-} \cup \Omega^{+}} U^{\prime}(c-v) R(v) \varphi_{\epsilon_{t}, \epsilon_{x}} \mathrm{~d} y \mathrm{~d} \tau-\int_{\Gamma} \llbracket(G(c, v), U(c-v)) \cdot \mathbf{n} \rrbracket \varphi_{\epsilon_{t}, \epsilon_{x}} \mathrm{~d} \gamma \\
\leq \int_{\Omega^{-} \cup \Omega^{+}}|R(v)| \varphi_{\epsilon_{t}, \epsilon_{x}} \mathrm{~d} y \mathrm{~d} \tau+\int_{\Gamma} \mathcal{R}(v) \varphi_{\epsilon_{t}, \epsilon_{x}} \mathrm{~d} \gamma
\end{array}
$$

Hence

$$
\Lambda_{U}^{\epsilon_{x}, \epsilon_{t}}(v) \leq\|R(v)\|_{L^{1}(\mathbb{R} \times(0, T) \backslash \Gamma)}+\|\mathcal{R}(v)\|_{L^{1}(\Gamma)},
$$

and the result follows. This completes the proof.

Proof of Proposition 7.2 (characterization of piecewise smooth entropy solutions). Let $v$ be any function satisfying the conditions (i) and (ii). Then, by Corollary 7.1 ,

$$
\|u(T)-v(T)\|_{L^{1}} \leq \int_{0}^{T} \mathcal{R}(v)(x(t), t) \mathrm{d} t .
$$

Next we show that $\mathcal{R}(v)=0$ if and only if conditions (iii) and (iv) are satisfied. This implies that $u=v$, as claimed.

So, by definition, $\mathcal{R}(v)=0$ on $\Gamma$ if

$$
-\llbracket(G(v, c), U(v-c)) \cdot \mathbf{n} \rrbracket \leq 0
$$

for all $c \in \mathbb{R}$ on each point of $\Gamma$. From this inequality, and since

$$
\mathbf{n}^{ \pm}= \pm \rho\left(-1, \frac{\mathrm{d} x}{\mathrm{~d} t}\right) \quad \text { where } \quad \rho^{-1}=\left|\left(-1, \frac{\mathrm{d} x}{\mathrm{~d} t}\right)\right|,
$$

we get that

$$
\begin{array}{ll}
\left(f\left(v^{+}\right)-f\left(v^{-}\right)\right)-\frac{\mathrm{d} x}{\mathrm{~d} t}\left(v^{+}-v^{-}\right) \leq 0 & \text { for } c<a=\min \left\{v^{-}, v^{+}\right\} \\
\left(f\left(v^{+}\right)-f\left(v^{-}\right)\right)-\frac{\mathrm{d} x}{\mathrm{~d} t}\left(v^{+}-v^{-}\right) \geq 0 & \text { for } c>b=\max \left\{v^{-}, v^{+}\right\} .
\end{array}
$$

These two inequalities are equivalent to condition (iii).

Finally, for $c \in(a, b)$ we get

$$
\operatorname{sign}\left(v^{+}-v^{-}\right)\left(\left(f\left(v^{+}\right)-2 f(c)+f\left(v^{-}\right)\right)-\frac{\mathrm{d} x_{i}}{\mathrm{~d} t}\left(v^{+}-2 c+v^{-}\right)\right) \leq 0 .
$$


After a few simple algebraic manipulations, we obtain the inequality

$$
\frac{f\left(v^{+}\right)-f\left(v^{-}\right)}{v^{+}-v^{-}} \leq \frac{f(c)-f\left(v^{-}\right)}{c-v^{-}} \quad \forall c \in(a, b),
$$

which is equivalent to condition (iv). This completes the proof.

Proof of Theorem 7.3 (characterization of the entropy solution). Let $u_{\nu}$ be the solution of the parabolic problem (4.1) and (4.2). Then

$\mathrm{E}\left(u_{0}, c ; u_{\nu}, \varphi\right)=-\nu \int_{0}^{T} \int_{\mathbb{R}^{d}} U^{\prime}\left(c-u_{\nu}\right) \Delta u_{\nu} \varphi \mathrm{d} y \mathrm{~d} t \leq \nu\left|u_{\nu}\right|_{L^{1}(T V)}\|\nabla \varphi\|_{L^{\infty}}$,

and by Proposition 5.2,

$$
\mathrm{E}\left(u_{0}, c ; u, \varphi\right)=\lim _{\nu \downarrow 0} \mathrm{E}\left(u_{0}, c ; u_{\nu}, \varphi\right) \leq 0 .
$$

This shows that the set of functions satisfying the inequality (7.3) is not empty.

Now, let $v$ be a function such that

$$
\mathrm{E}\left(u_{0}, c ; v, \varphi\right) \leq 0 .
$$

Then, by Theorem 6.2 we have

$$
|u(T)-v(T)|_{U} \leq 2 \inf _{\epsilon_{x}, \epsilon_{t}>0}\left(\mathrm{~A} \epsilon_{x}+\mathrm{C} \epsilon_{t}+\Lambda_{U}^{\epsilon_{x}, \epsilon_{t}}\right),
$$

where

$$
\Lambda_{U}^{\epsilon_{x}, \epsilon_{t}}=\frac{1}{\Phi_{\epsilon_{t}}(T)} \int_{0}^{T} \int_{\mathbb{R}^{d}} \sup _{c \in \mathbb{R}} E_{U}^{\epsilon_{x}, \epsilon_{t}}(v, c ; x, t) \mathrm{d} x \mathrm{~d} t
$$

Since

$$
E_{U}^{\epsilon_{x}, \epsilon_{t}}(v, c ; x, t)=E_{\mathrm{small}}+E_{\mathrm{diss}}
$$

where

$$
\begin{aligned}
E_{\text {small }} & =-\int_{0}^{T} \int_{\mathbb{R}^{d}} U^{\prime}(c-v)(r(x, t)-r(y, \tau)) \varphi_{\epsilon_{t}, \epsilon_{x}} \mathrm{~d} y \mathrm{~d} \tau \\
E_{\text {diss }} & =\mathrm{E}\left(u_{0}, c ; v, \varphi_{\epsilon_{x}, \epsilon_{t}}\right)
\end{aligned}
$$

we have that

$$
\begin{aligned}
E_{\text {small }} & \leq \int_{0}^{T} \int_{\mathbb{R}^{d}}|r(x, t)-r(y, \tau)| \varphi_{\epsilon_{t}, \epsilon_{x}} \mathrm{~d} y \mathrm{~d} \tau \\
E_{\text {diss }} & \leq 0
\end{aligned}
$$

and so

$$
\Lambda_{U}^{\epsilon_{x}, \epsilon_{t}} \leq c_{1} \epsilon_{x}|r|_{L^{1}(T V)}+\epsilon_{t}\left|r_{t}\right|_{L^{1}\left(L^{1}\right)}
$$

Minimizing over the parameters $\epsilon_{t}$ and $\epsilon_{x}$, we see that $v$ is in fact the entropy solution $u$. This completes the proof. 